Supporting Information to

\title{
Novel Sesquiterpene Skeletons by Multiple Wagner-Meerwein Rearrangements of a Longipinane-1,9-diol Derivative
}

Concepción Armenta-Salinas, ${ }^{*},{ }^{\dagger}$ Ramón Guzmán-Mejía, ${ }^{\dagger}$ Hugo A. García-Gutiérrez, ${ }^{\dagger}$ Luisa

U. Román-Marín, ${ }^{\dagger}$ Juan D. Hernández-Hernández, ${ }^{\dagger}$ Carlos M. Cerda-García-Rojas, ${ }^{*}{ }^{\star}$ and Pedro Joseph-Nathan

†Instituto de Investigaciones Químico Biológicas, Universidad Michoacana de San Nicolás de Hidalgo, Ciudad Universitaria, Morelia, Michoacán, 58030 Mexico

†Departamento de Química, Centro de Investigación y de Estudios Avanzados del Instituto Politécnico Nacional, Apartado 14-740, Mexico City, 07000 Mexico 


\section{Contents}

Figure S1. ${ }^{1} \mathrm{H}$ and ${ }^{13} \mathrm{C}$ NMR spectra of rasteviol 7, 7-1-d, and 7-1,9- $d_{2}$ in $\mathrm{CDCl}_{3}$.

Figure S2. ${ }^{1} \mathrm{H}$ and ${ }^{13} \mathrm{C}$ NMR spectra of 8, 8- $1-d$, and 8- $1,9-d_{2}$ in $\mathrm{CDCl}_{3}$.

Figure S3. ${ }^{1} \mathrm{H}$ and ${ }^{13} \mathrm{C}$ NMR spectra of 9 in $\mathrm{CDCl}_{3}$.

Figure S4 COSY spectra of $\mathbf{9}$ in $\mathrm{CDCl}_{3}$.

Figure S5. ${ }^{1} \mathrm{H}$ and ${ }^{13} \mathrm{C}$ NMR spectra of $\mathbf{1 0}$ in $\mathrm{CDCl}_{3}$.

Figure S6. ${ }^{1} \mathrm{H}$ and ${ }^{13} \mathrm{C}$ NMR spectra of 11, 11- $1-d$, and 11-1,9- $d_{2}$ in $\mathrm{CDCl}_{3} . \quad$ S8

Figure S7. ${ }^{1} \mathrm{H}$ and ${ }^{13} \mathrm{C}$ NMR spectra of $\mathbf{1 2}$ in $\mathrm{CDCl}_{3}$.

$\begin{array}{ll}\text { Figure S8 COSY spectra of } \mathbf{1 2} \text { in } \mathrm{CDCl}_{3} . & \mathrm{S} 10\end{array}$

Figure S9. ${ }^{1} \mathrm{H}$ and ${ }^{13} \mathrm{C}$ NMR spectra of $\mathbf{1 3}$ in $\mathrm{CDCl}_{3}$. $\quad \mathrm{S} 11$

Figure S10. ${ }^{1} \mathrm{H}$ and ${ }^{13} \mathrm{C}$ NMR spectra of $\mathbf{1 4}, \mathbf{1 4}-1-d$, and 14-1,9- $d_{2}$ in $\mathrm{CDCl}_{3} . \quad$ S12

Figure S11. ${ }^{1} \mathrm{H}$ and ${ }^{13} \mathrm{C}$ NMR spectra of $\mathbf{1 5}$ in DMSO- $d_{6}$. S13

$\begin{array}{ll}\text { Figure S12. }{ }^{1} \mathrm{H} \text { and }{ }^{13} \mathrm{C} \text { NMR spectra of } \mathbf{1 6} \text { in } \mathrm{CDCl}_{3} . & \mathrm{S} 14\end{array}$

$\begin{array}{lr}\text { Figure S13 COSY spectra of } \mathbf{1 6} \text { in } \mathrm{CDCl}_{3} . & \mathrm{S} 15\end{array}$

Figure S14. ${ }^{1} \mathrm{H}$ and ${ }^{13} \mathrm{C}$ NMR spectra of $\mathbf{1 7}$ in $\mathrm{CDCl}_{3}$. $\quad \mathrm{S} 16$

Figure S15. ${ }^{1} \mathrm{H}$ and ${ }^{13} \mathrm{C}$ NMR spectra of $\mathbf{1 8}, \mathbf{1 8}-1-d$, and 18-1,9- $d_{2}$ in $\mathrm{CDCl}_{3} . \quad$ S17

Figure S16. ${ }^{1} \mathrm{H}$ and ${ }^{13} \mathrm{C}$ NMR spectra of $\mathbf{1 9}$ in $\mathrm{CDCl}_{3}$. $\quad \mathrm{S} 18$

Figure S17. ${ }^{1} \mathrm{H}$ and ${ }^{13} \mathrm{C}$ NMR spectra of $\mathbf{2 0}$ in $\mathrm{CDCl}_{3} . \quad \mathrm{S} 19$

Figure S18. ${ }^{1} \mathrm{H}$ and ${ }^{13} \mathrm{C}$ NMR spectra of 21 in $\mathrm{CDCl}_{3}$. $\quad$ S20

Figure S19. ${ }^{1} \mathrm{H}$ and ${ }^{13} \mathrm{C}$ NMR spectra of 22 in $\mathrm{CDCl}_{3}$. $\quad$ S21

Table S1. Single-crystal X-ray data for compounds 10, 15, 20, and 21................S22 

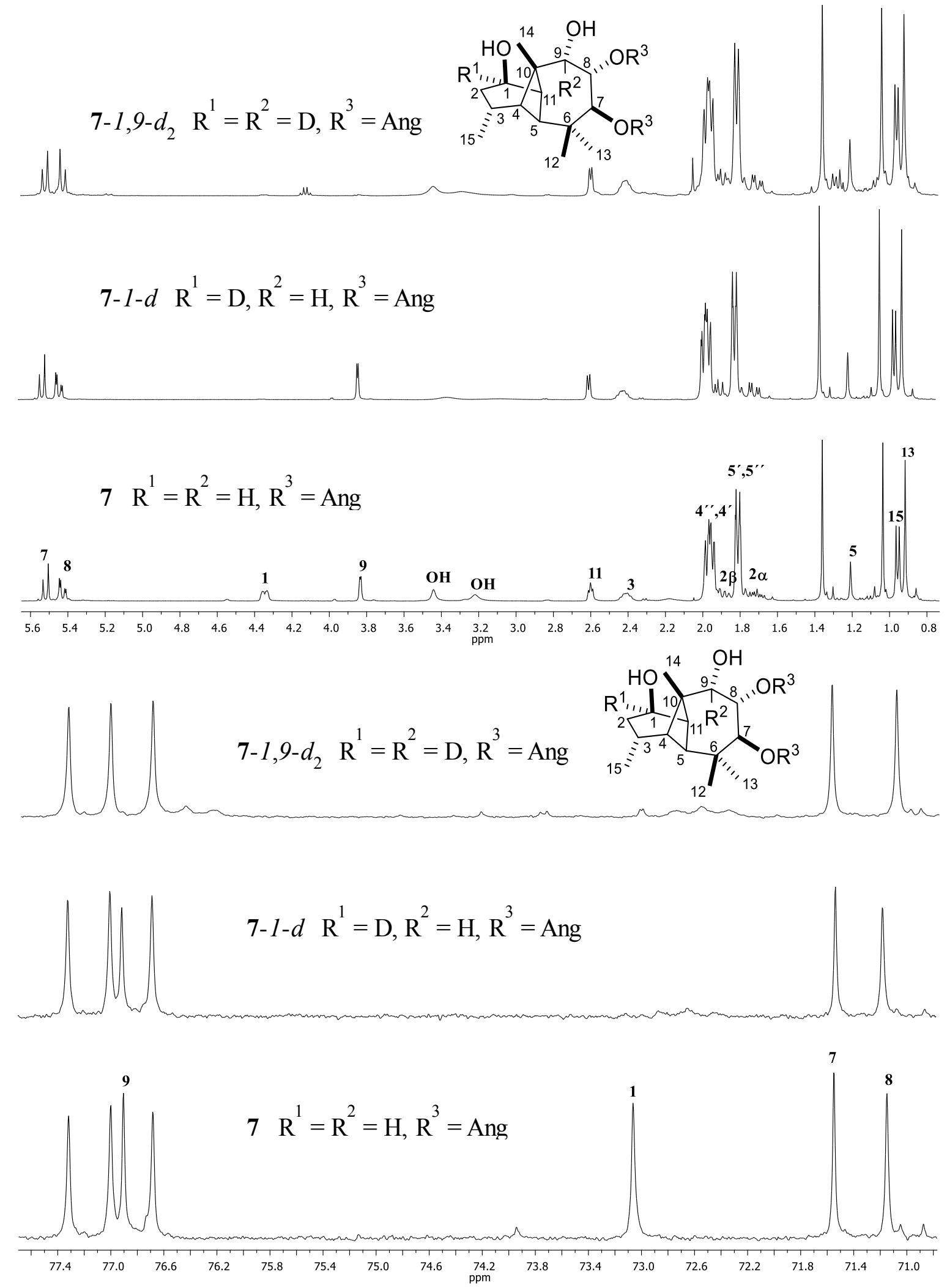

Figure S1. ${ }^{1} \mathrm{H}(400 \mathrm{MHz})$ and ${ }^{13} \mathrm{C}(100 \mathrm{MHz}) \mathrm{NMR}$ spectra of 7, 7-1-d, and 7-1,9- $d_{2}$ in $\mathrm{CDCl}_{3}$. 

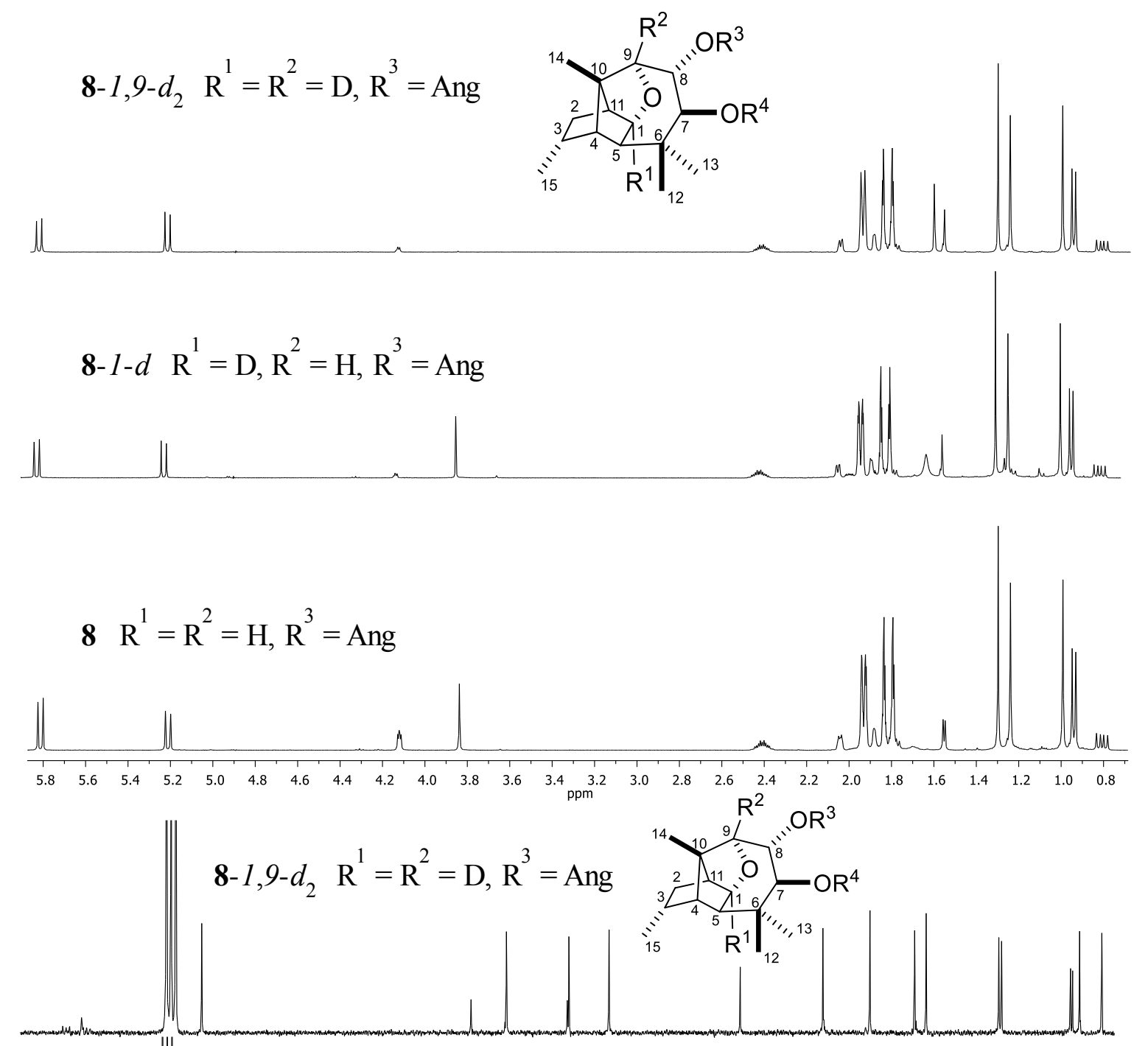

8- $1-d \quad \mathrm{R}^{1}=\mathrm{D}, \mathrm{R}^{2}=\mathrm{H}, \mathrm{R}^{3}=$ Ang
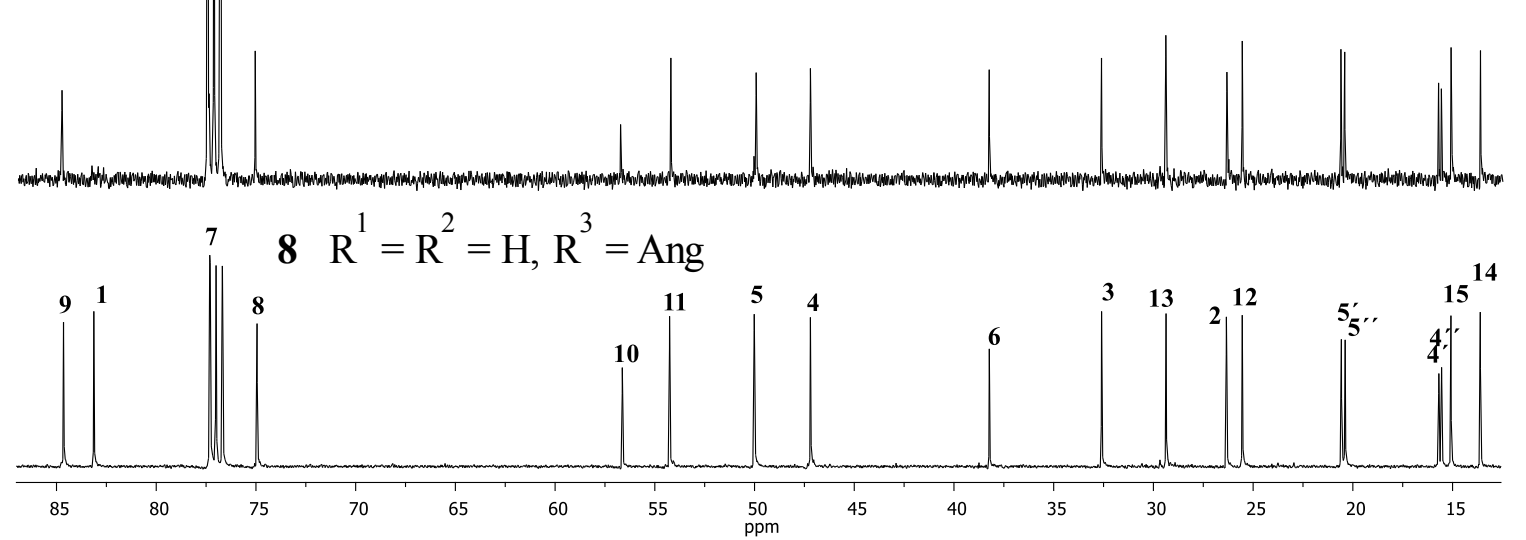

Figure S2. ${ }^{1} \mathrm{H}(400 \mathrm{MHz})$ and ${ }^{13} \mathrm{C}(100 \mathrm{MHz}) \mathrm{NMR}$ spectra of 8, 8-1-d, and 8-1,9- $d_{2}$ in $\mathrm{CDCl}_{3}$. 

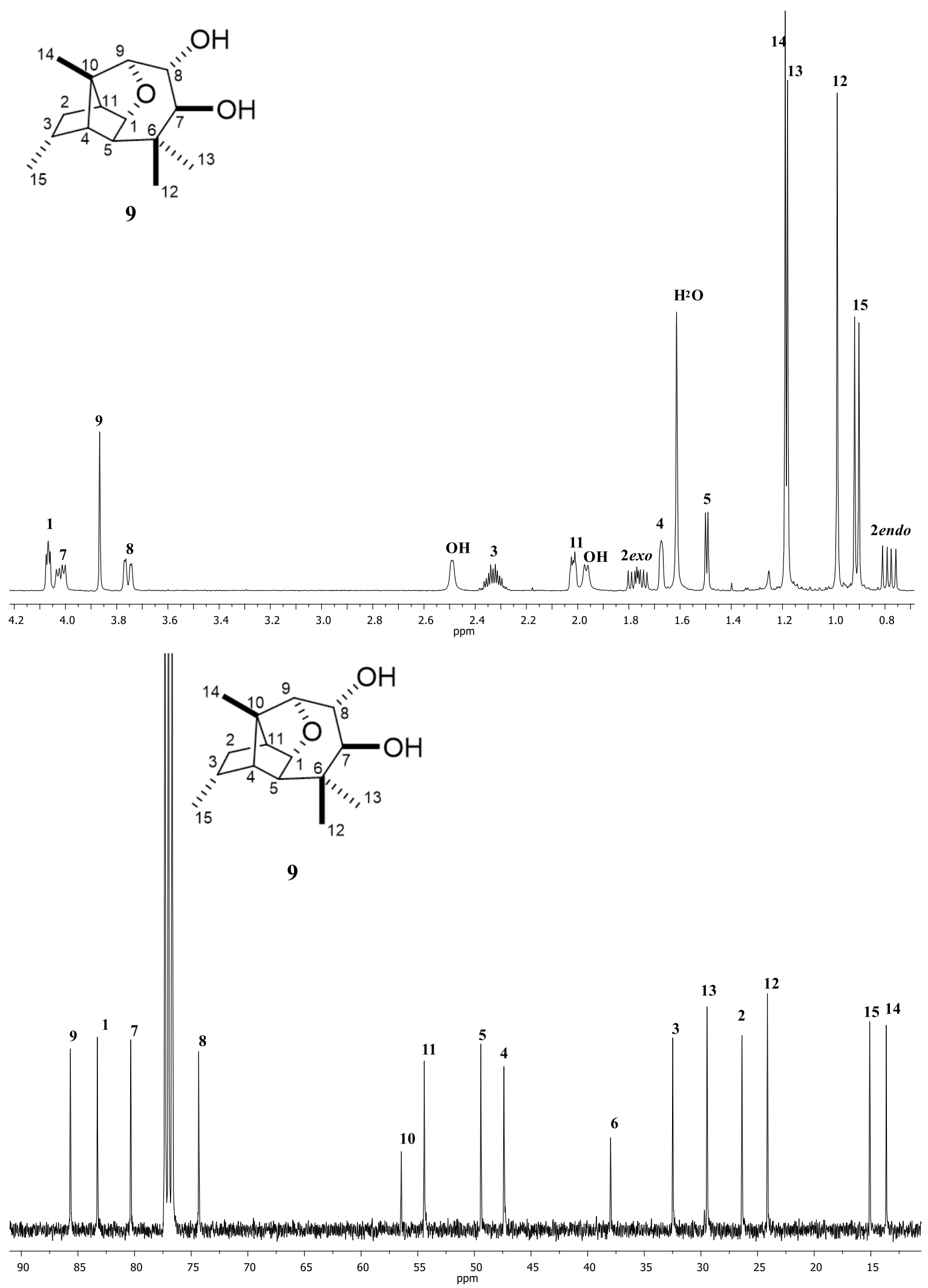

Figure S3. ${ }^{1} \mathrm{H}(400 \mathrm{MHz})$ and ${ }^{13} \mathrm{C}(100 \mathrm{MHz}) \mathrm{NMR}$ spectra of 9 in $\mathrm{CDCl}_{3}$. 


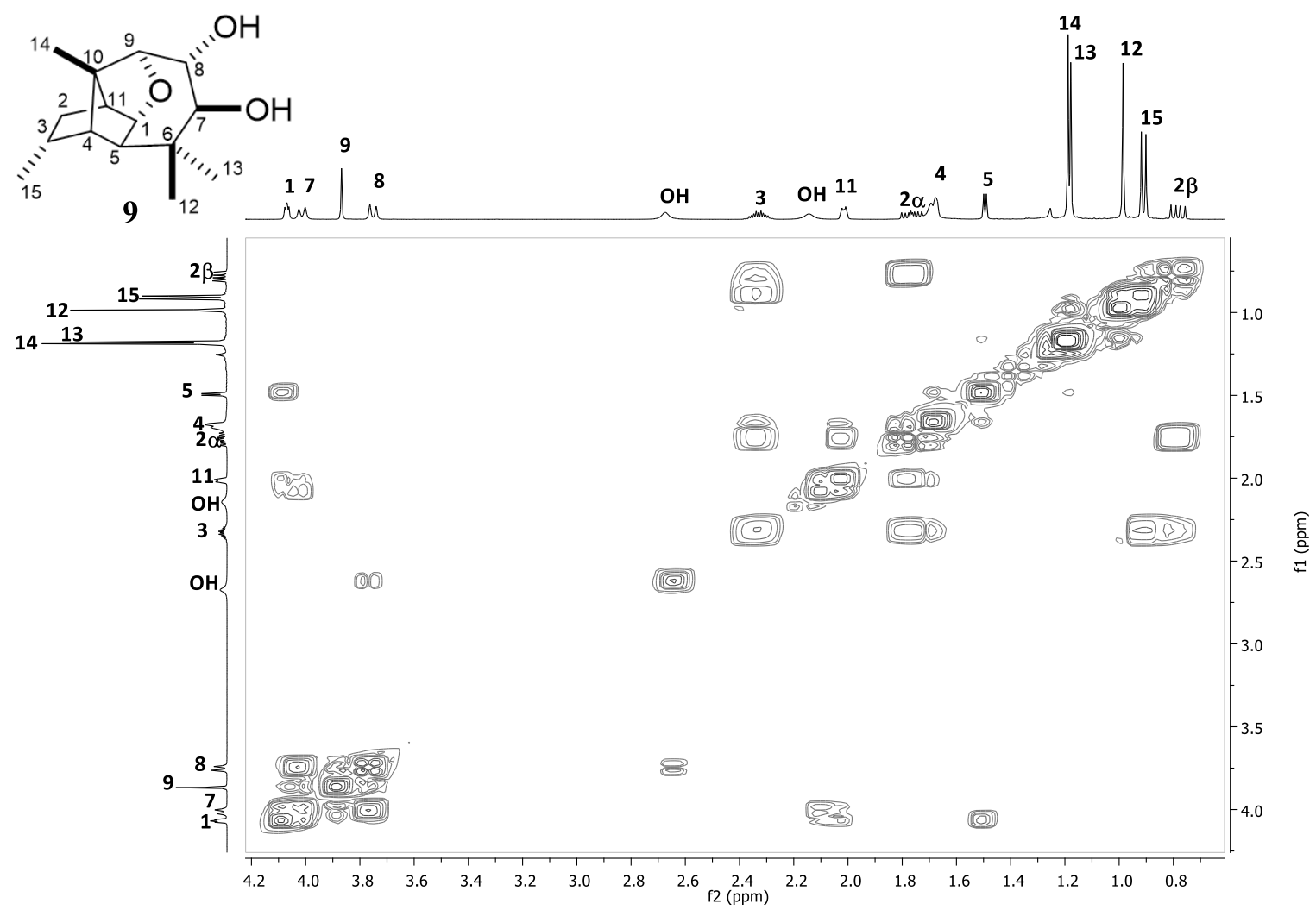

Figure S4. COSY spectra of 9 in $\mathrm{CDCl}_{3}$. 

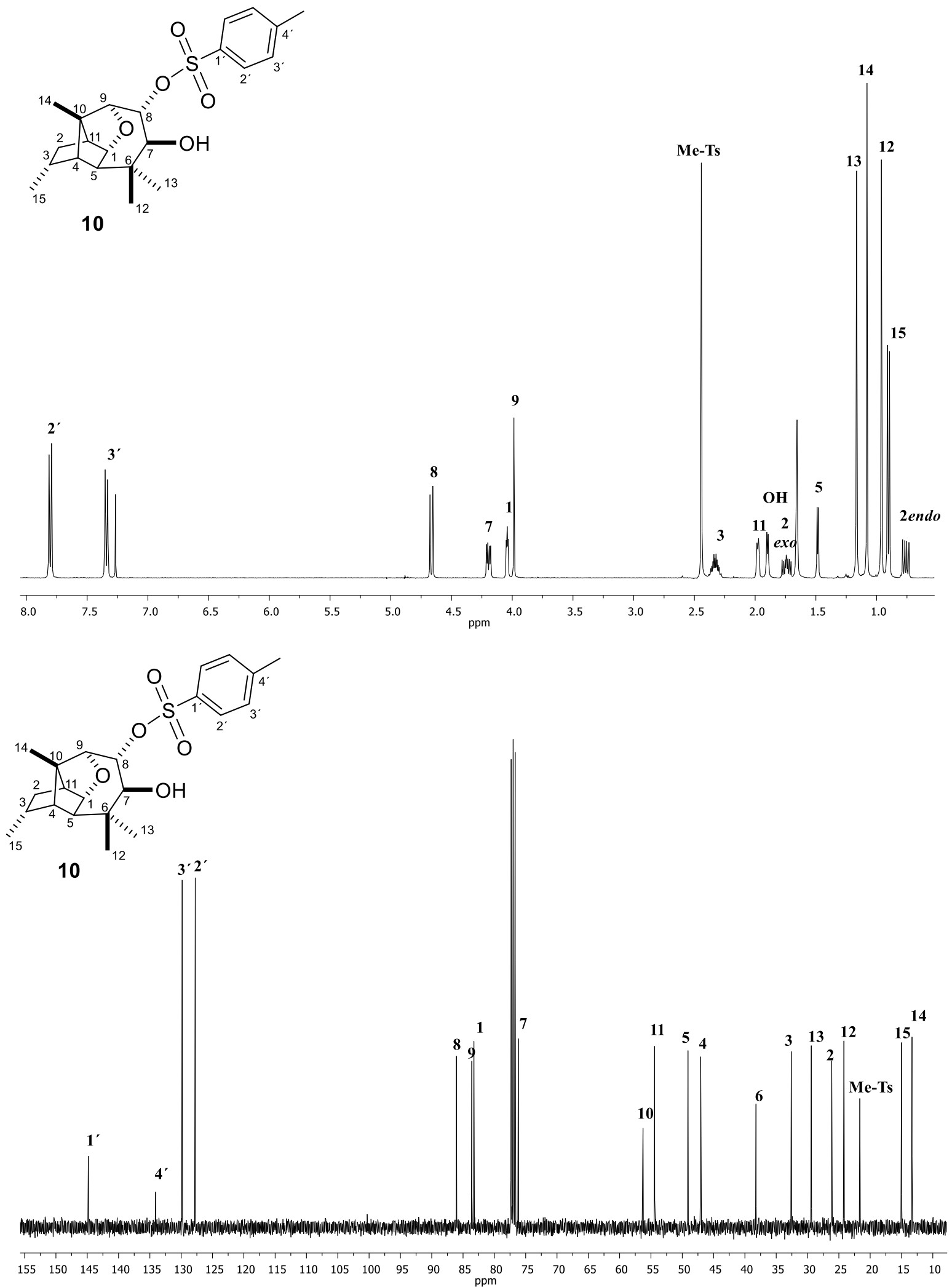

Figure S5. ${ }^{1} \mathrm{H}(400 \mathrm{MHz})$ and ${ }^{13} \mathrm{C}(100 \mathrm{MHz}) \mathrm{NMR}$ spectra of 10 in $\mathrm{CDCl}_{3}$. 


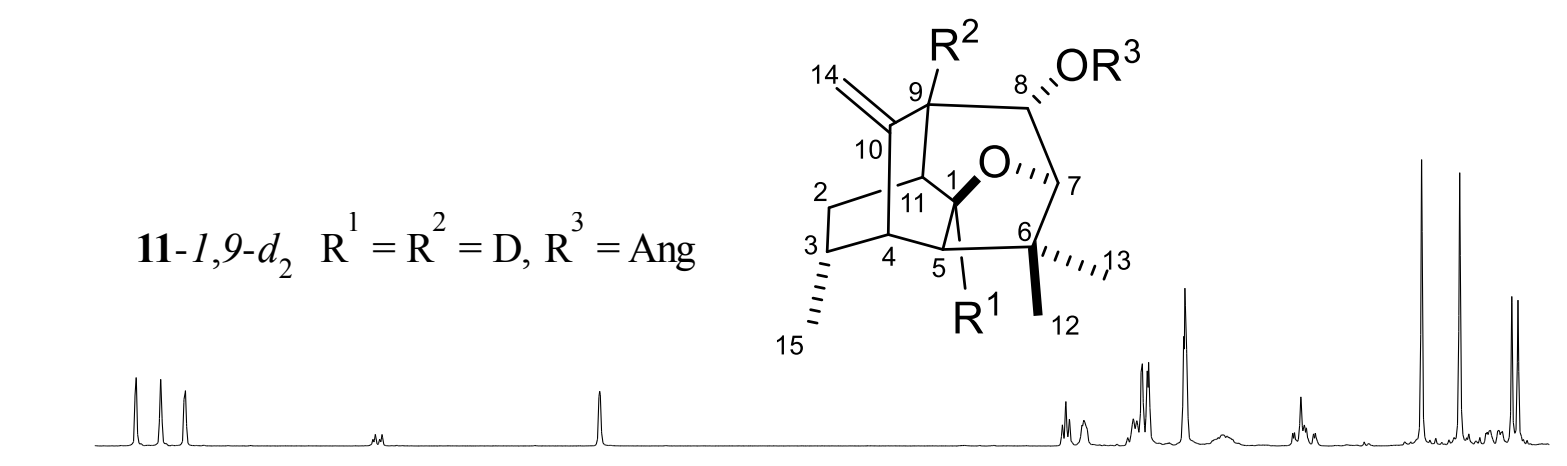

11-1-d $\mathrm{R}^{1}=\mathrm{D}, \mathrm{R}^{2}=\mathrm{H}, \mathrm{R}^{3}=$ Ang
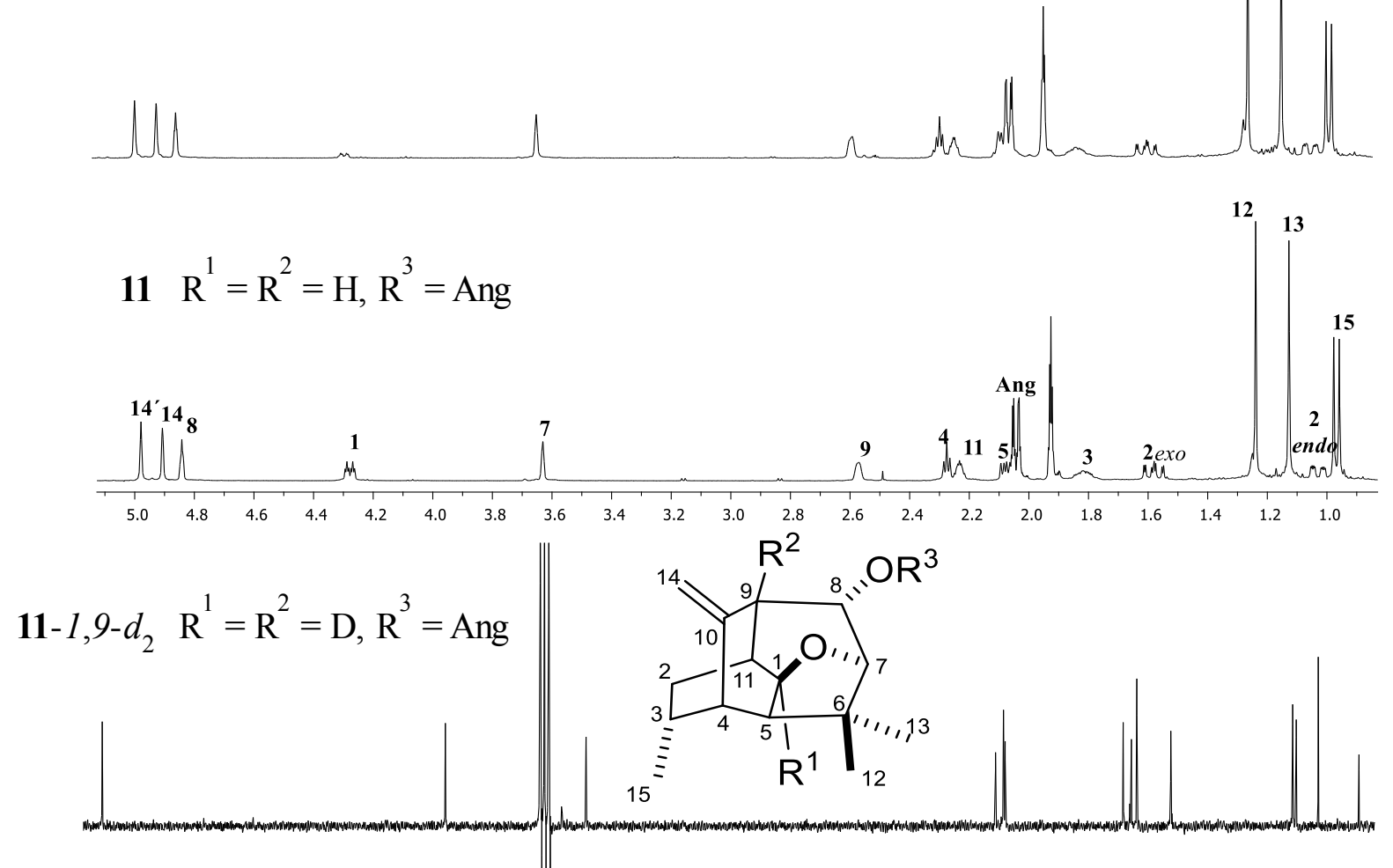

11-1-d $\mathrm{R}^{1}=\mathrm{D}, \mathrm{R}^{2}=\mathrm{H}, \mathrm{R}^{3}=\mathrm{Ang}$
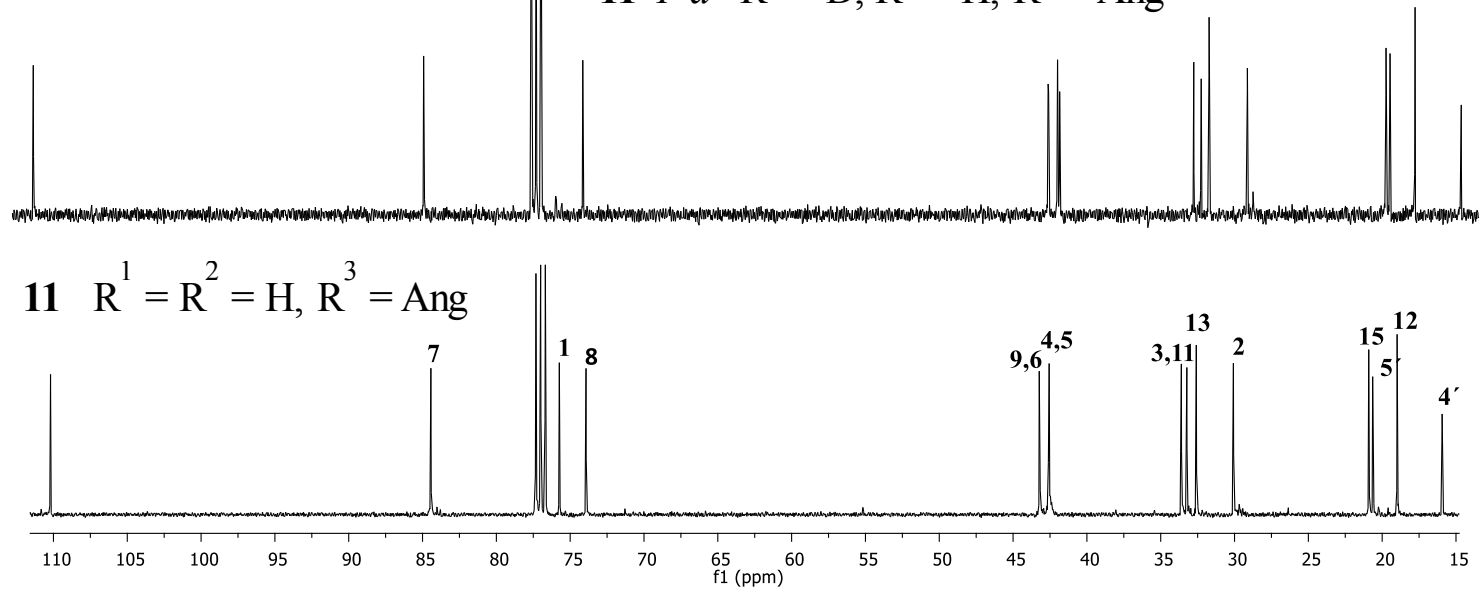

Figure S6. ${ }^{1} \mathrm{H}(400 \mathrm{MHz})$ and ${ }^{13} \mathrm{C}(100 \mathrm{MHz}) \mathrm{NMR}$ spectra of $11,11-1-d$, and 11-1,9- $d_{2}$ in $\mathrm{CDCl}_{3}$. 


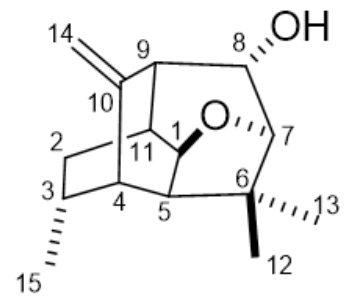

12
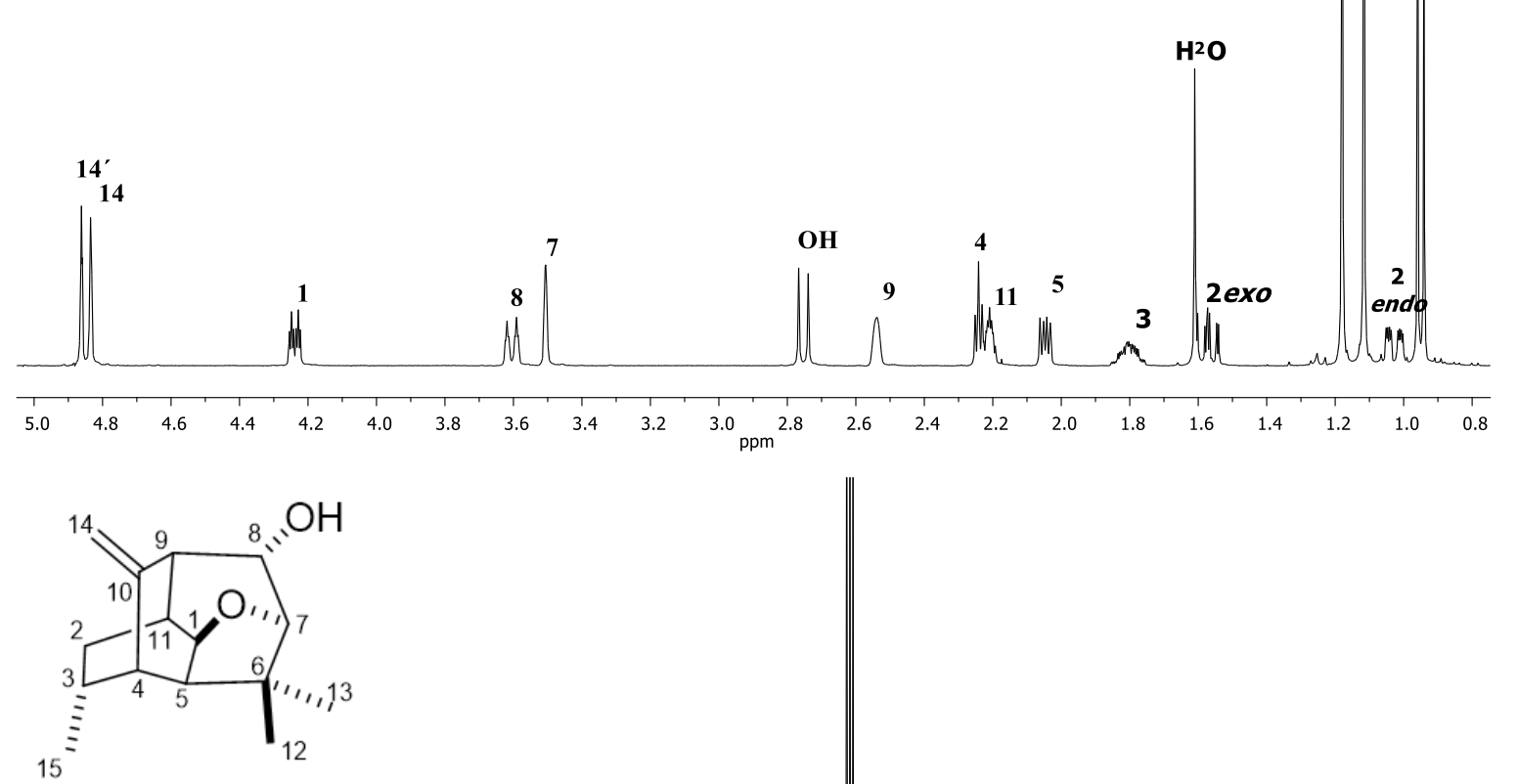

12

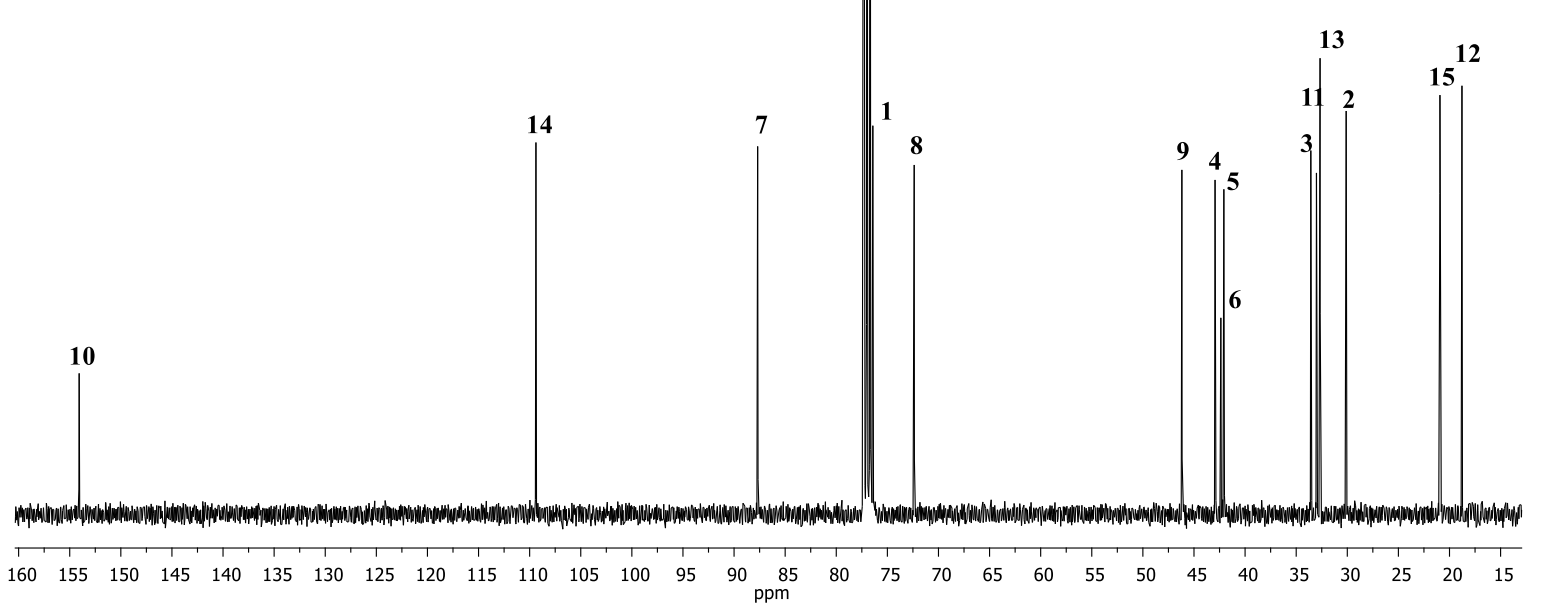

Figure S7. ${ }^{1} \mathrm{H}(400 \mathrm{MHz})$ and ${ }^{13} \mathrm{C}(100 \mathrm{MHz}) \mathrm{NMR}$ spectra of 12 in $\mathrm{CDCl}_{3}$. 

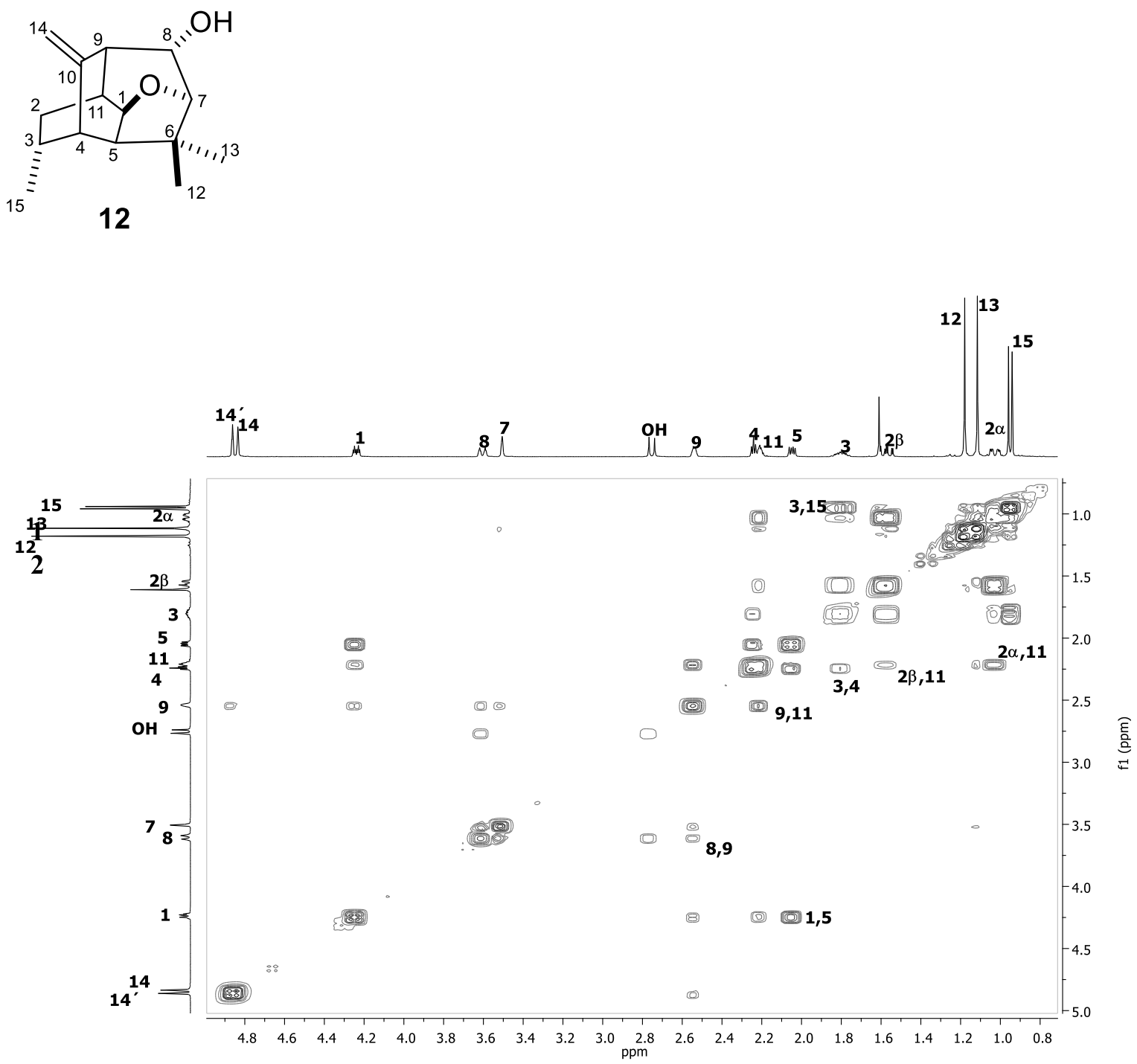

Figure S8. COSY spectra of 12 in $\mathrm{CDCl}_{3}$. 

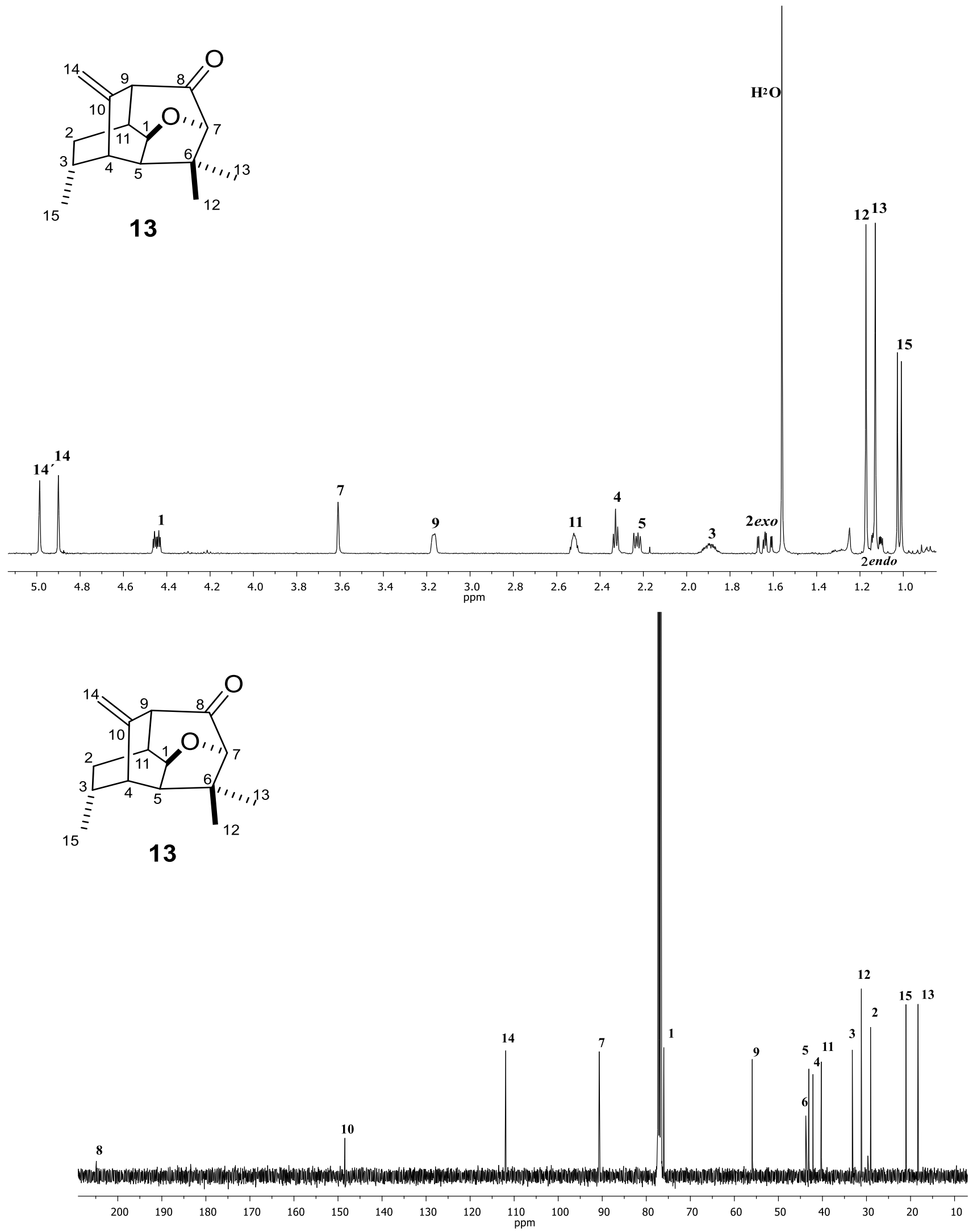

Figure 59. ${ }^{1} \mathrm{H}(400 \mathrm{MHz})$ and ${ }^{13} \mathrm{C}(100 \mathrm{MHz}) \mathrm{NMR}$ spectra of 13 in $\mathrm{CDCl}_{3}$. 
14-1,9- $d_{2} \quad \mathrm{R}^{1}=\mathrm{R}^{2}=\mathrm{D}, \mathrm{R}^{3}=$ Ang

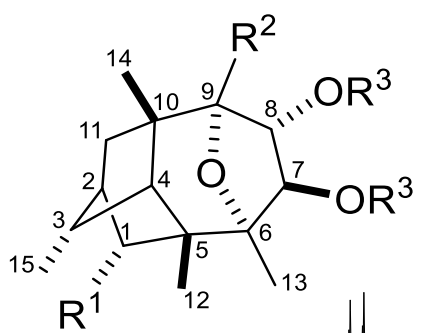

$\mu$

14- $1-d \quad \mathrm{R}^{1}=\mathrm{D}, \mathrm{R}^{2}=\mathrm{H}, \mathrm{R}^{3}=\mathrm{Ang}$

$14 \mathrm{R}^{1}=\mathrm{R}^{2}=\mathrm{H}, \mathrm{R}^{3}=\mathrm{Ang}$
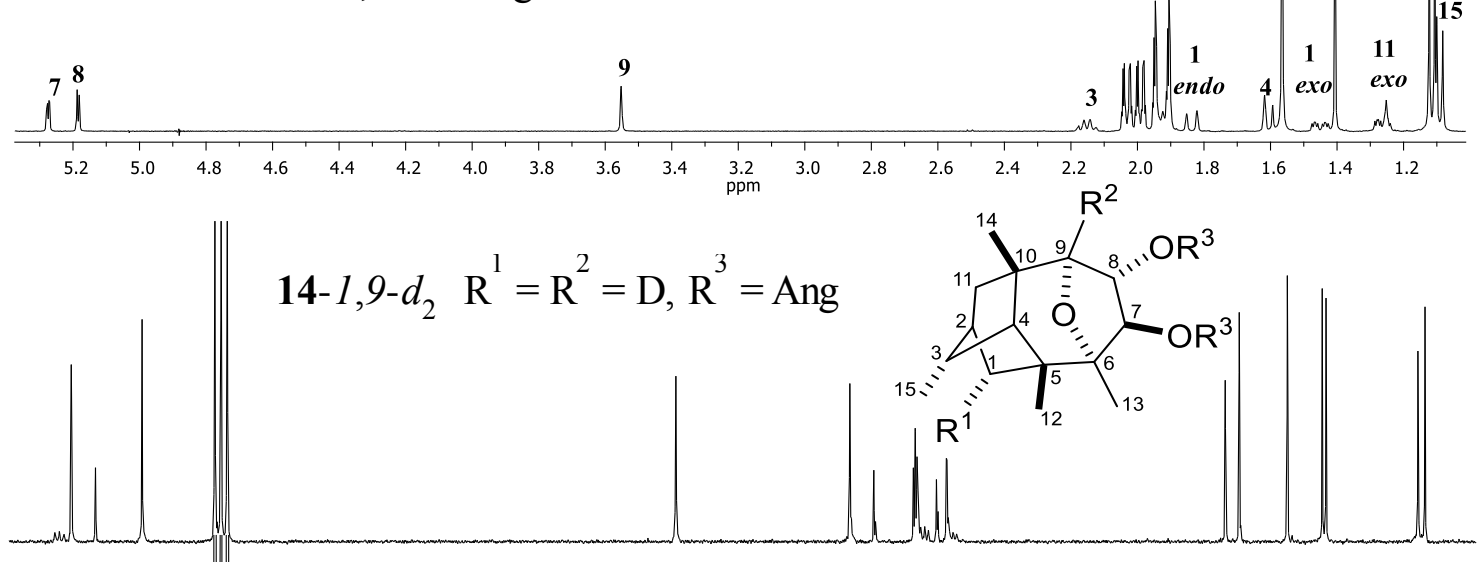

14- $1-d \quad \mathrm{R}^{1}=\mathrm{D}, \mathrm{R}^{2}=\mathrm{H}, \mathrm{R}^{3}=$ Ang
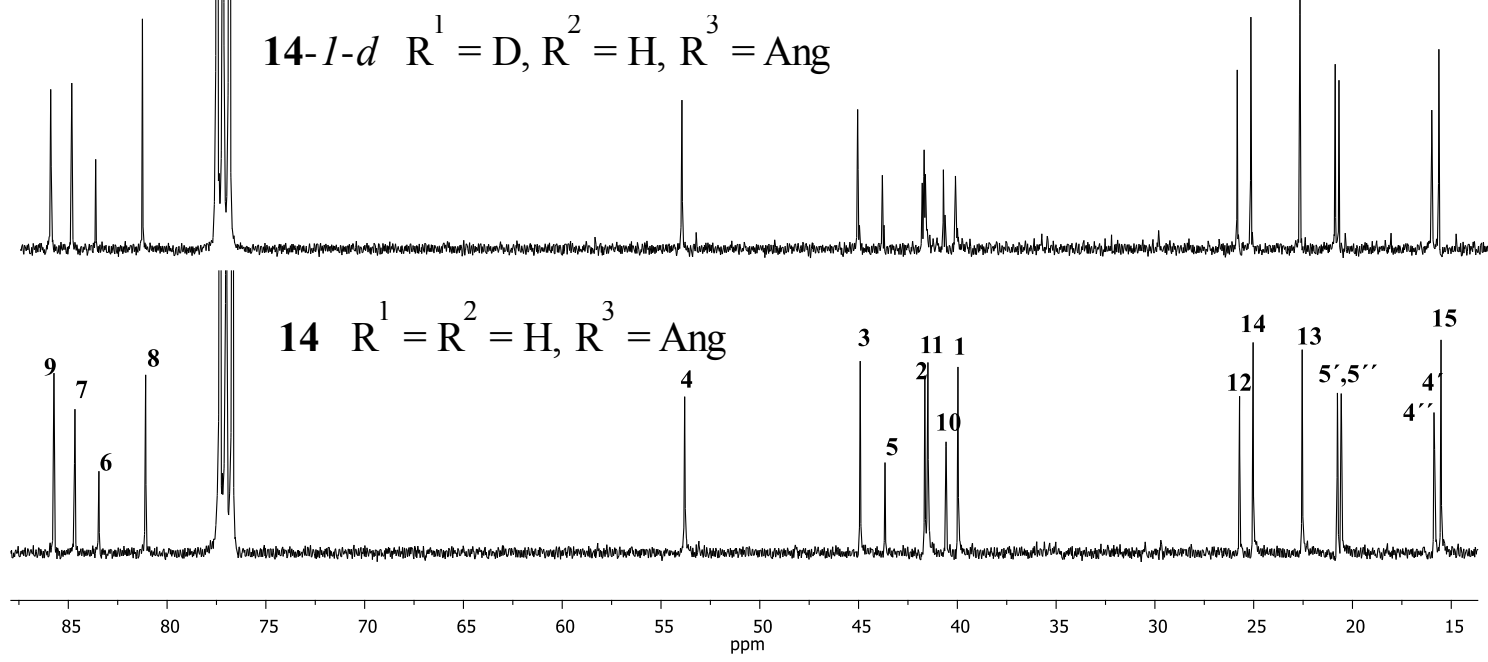

Figure S10. ${ }^{1} \mathrm{H}(400 \mathrm{MHz})$ and ${ }^{13} \mathrm{C}(100 \mathrm{MHz}) \mathrm{NMR}$ spectra of $14,14-1-d$, and $14-1,9-d_{2}$ in $\mathrm{CDCl}_{3}$. 


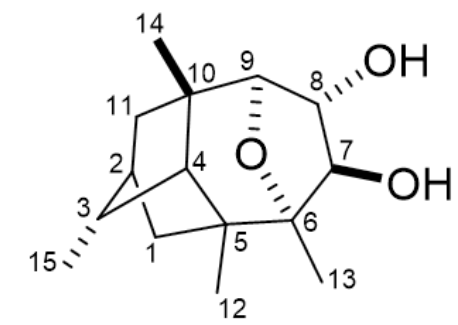

15

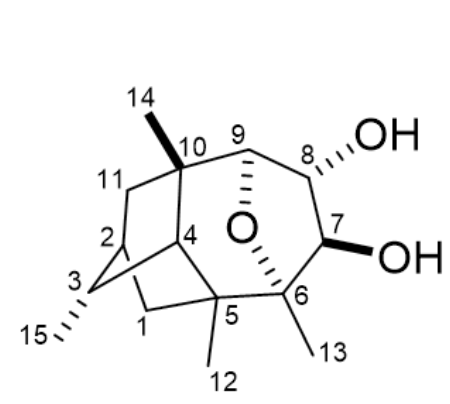

15
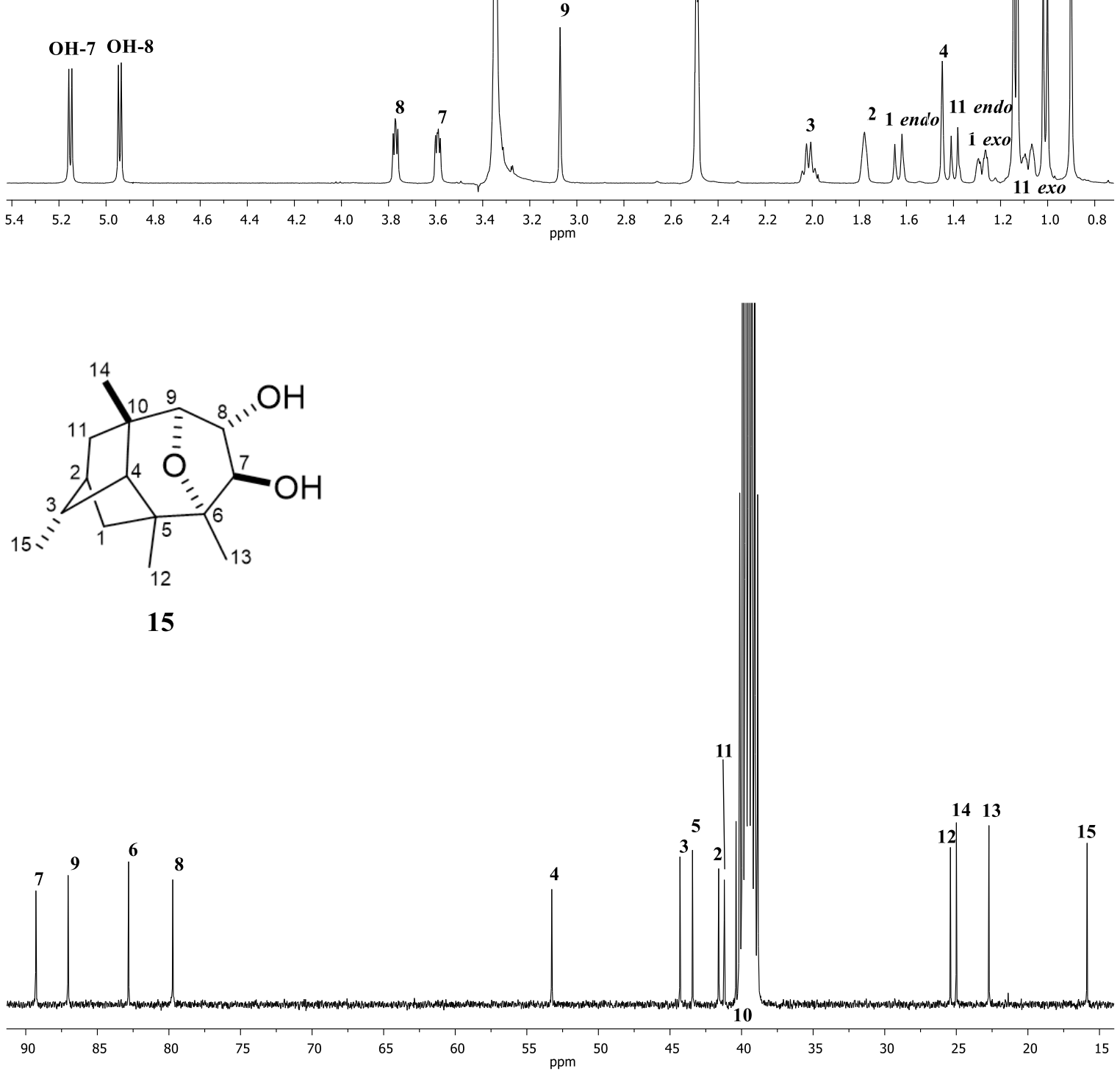

Figure S11. ${ }^{1} \mathrm{H}(400 \mathrm{MHz})$ and ${ }^{13} \mathrm{C}(100 \mathrm{MHz}) \mathrm{NMR}$ spectra of 15 in DMSO- $d_{6}$. 


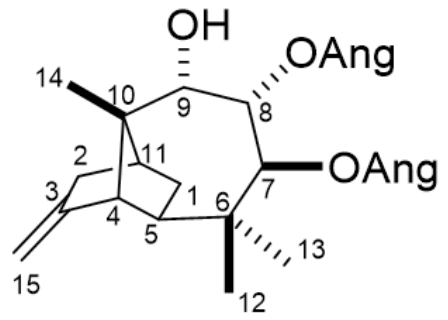

16
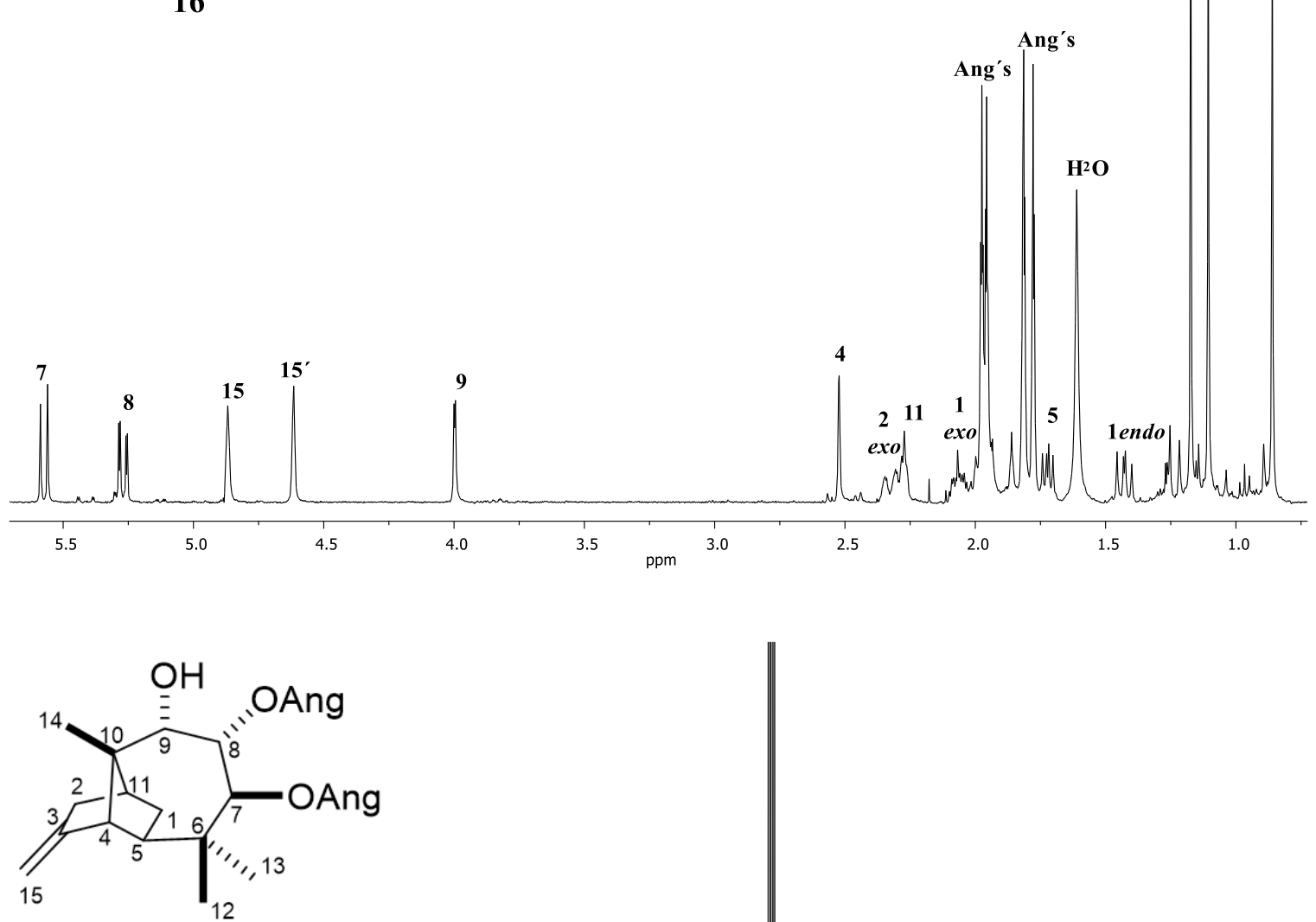

16

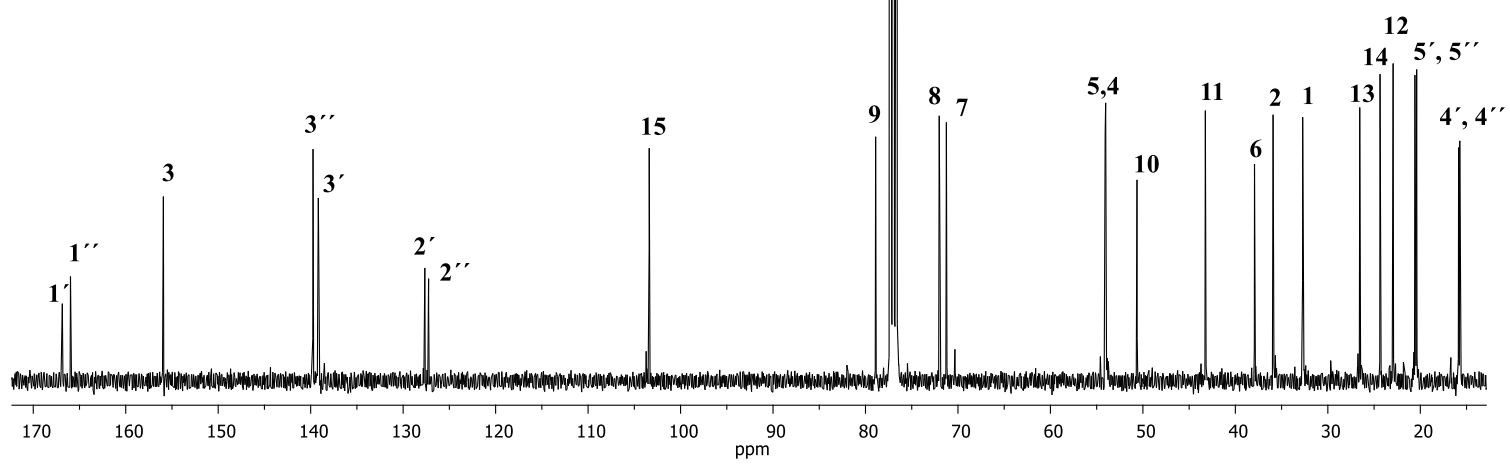

Figure S12. ${ }^{1} \mathrm{H}(400 \mathrm{MHz})$ and ${ }^{13} \mathrm{C}(100 \mathrm{MHz}) \mathrm{NMR}$ spectra of 16 in $\mathrm{CDCl}_{3}$. 


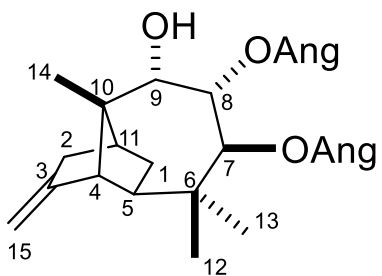

16

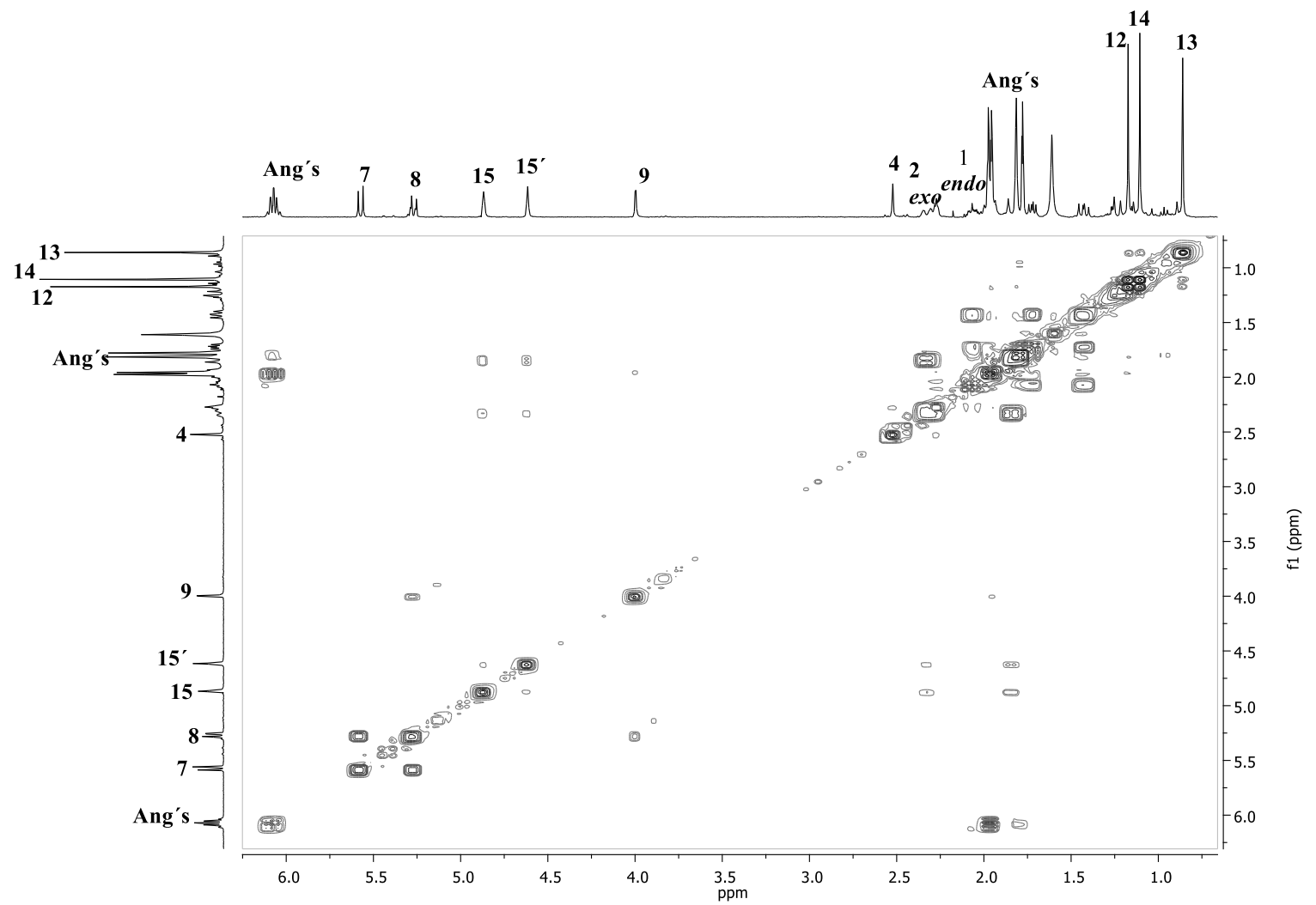

Figure S13. COSY spectra of 16 in $\mathrm{CDCl}_{3}$. 


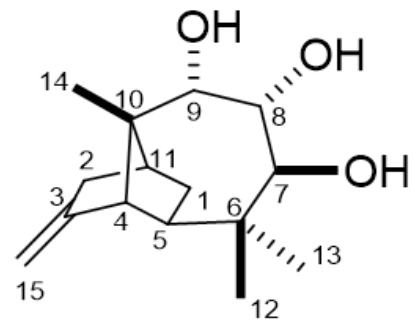

17
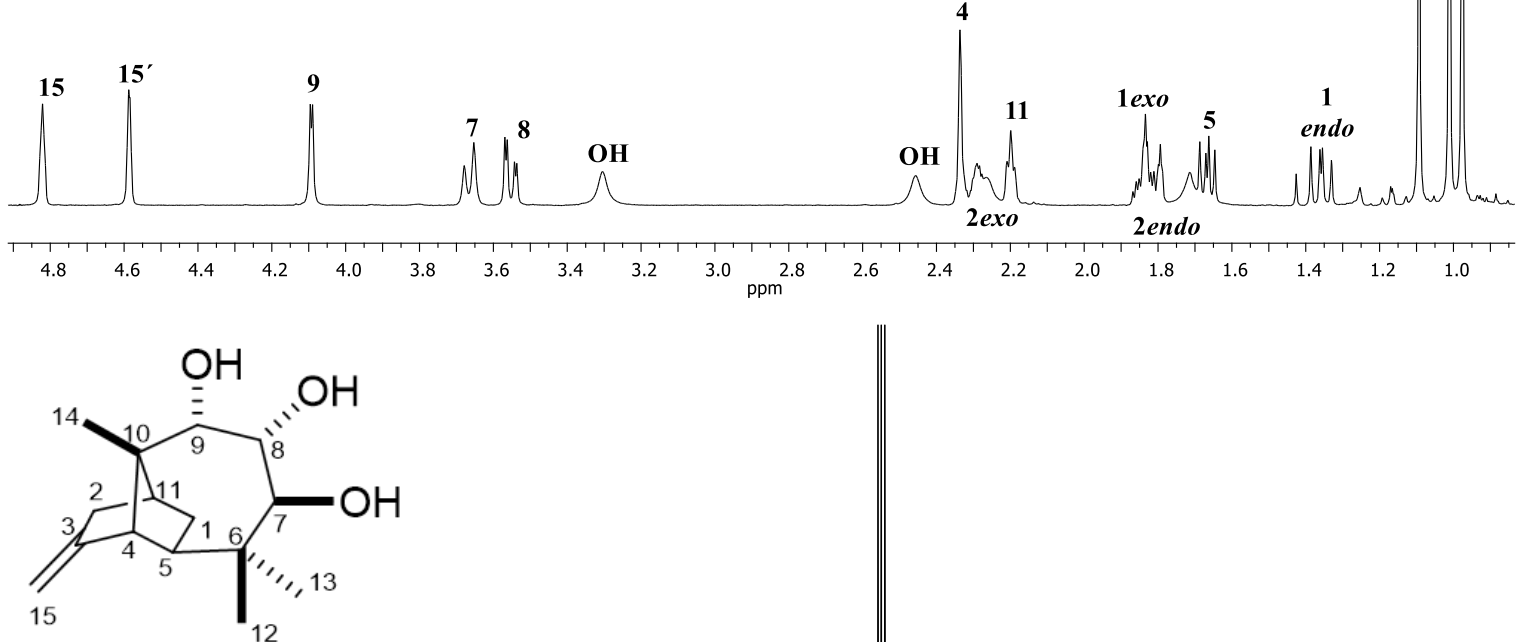

17
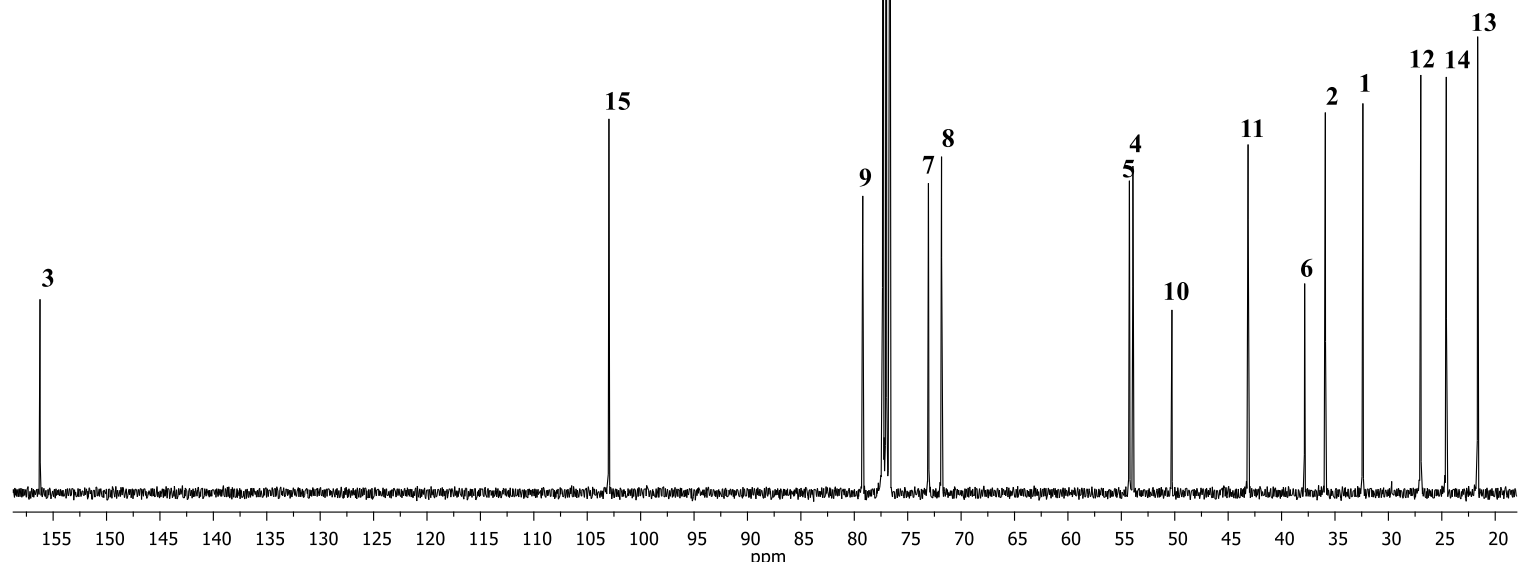

$\begin{array}{llllllllllllllllllllllllllllllllll}155 & 150 & 145 & 140 & 135 & 130 & 125 & 120 & 115 & 110 & 105 & 100 & 95 & 90 & 85 & 80 & 75 & 70 & 65 & 60 & 55 & 50 & 45 & 40 & 35 & 30 & 25 & 20\end{array}$

Figure S14. ${ }^{1} \mathrm{H}(400 \mathrm{MHz})$ and ${ }^{13} \mathrm{C}(100 \mathrm{MHz}) \mathrm{NMR}$ spectra of 17 in $\mathrm{CDCl}_{3}$. 
18-1,9- $d_{2} \quad R^{1}=R^{2}=D$

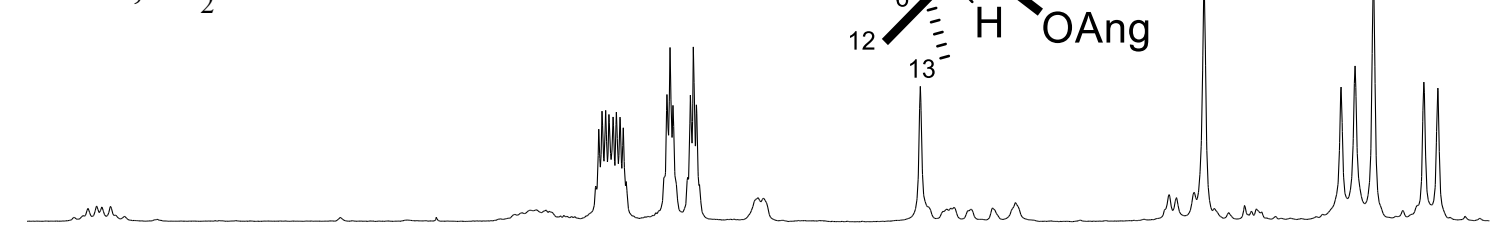

18- $1-d \mathrm{R}^{1}=\mathrm{D}, \mathrm{R}^{2}=\mathrm{H}$
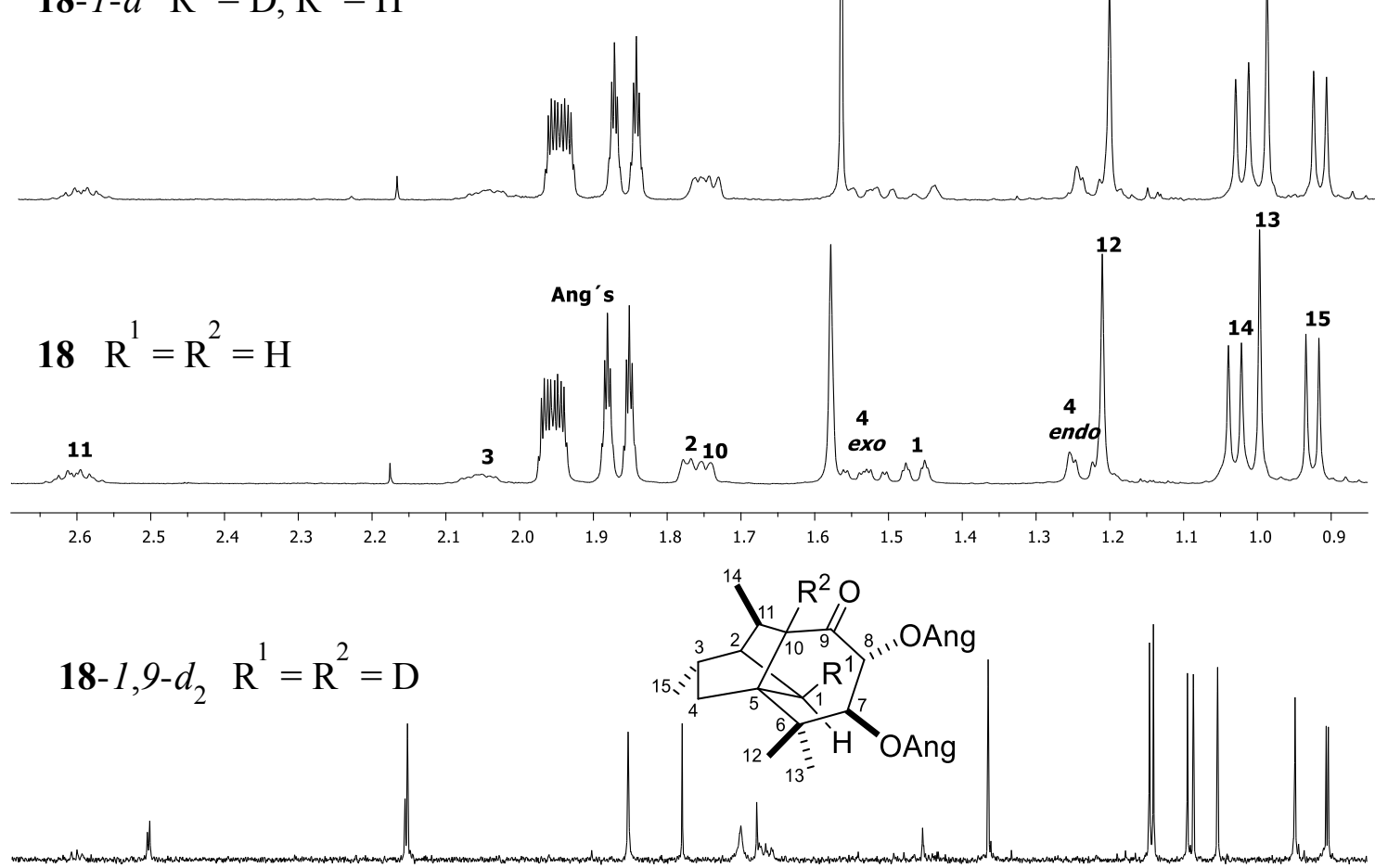

18- $1-d \quad \mathrm{R}^{1}=\mathrm{D}, \mathrm{R}^{2}=\mathrm{H}$
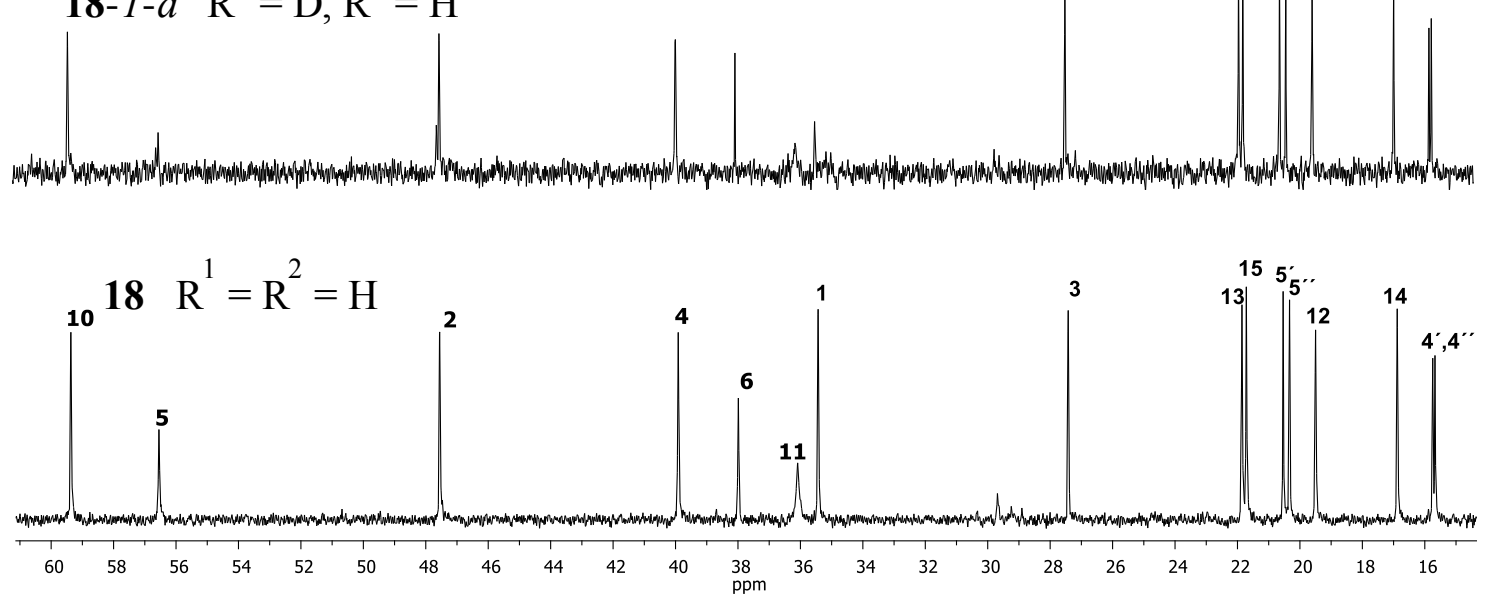

Figure S15. ${ }^{1} \mathrm{H}(400 \mathrm{MHz})$ and ${ }^{13} \mathrm{C}(100 \mathrm{MHz}) \mathrm{NMR}$ spectra of $18,18-1-d$, and $18-1,9-d_{2}$ in $\mathrm{CDCl}_{3}$. 


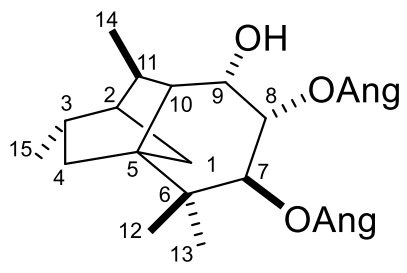

19
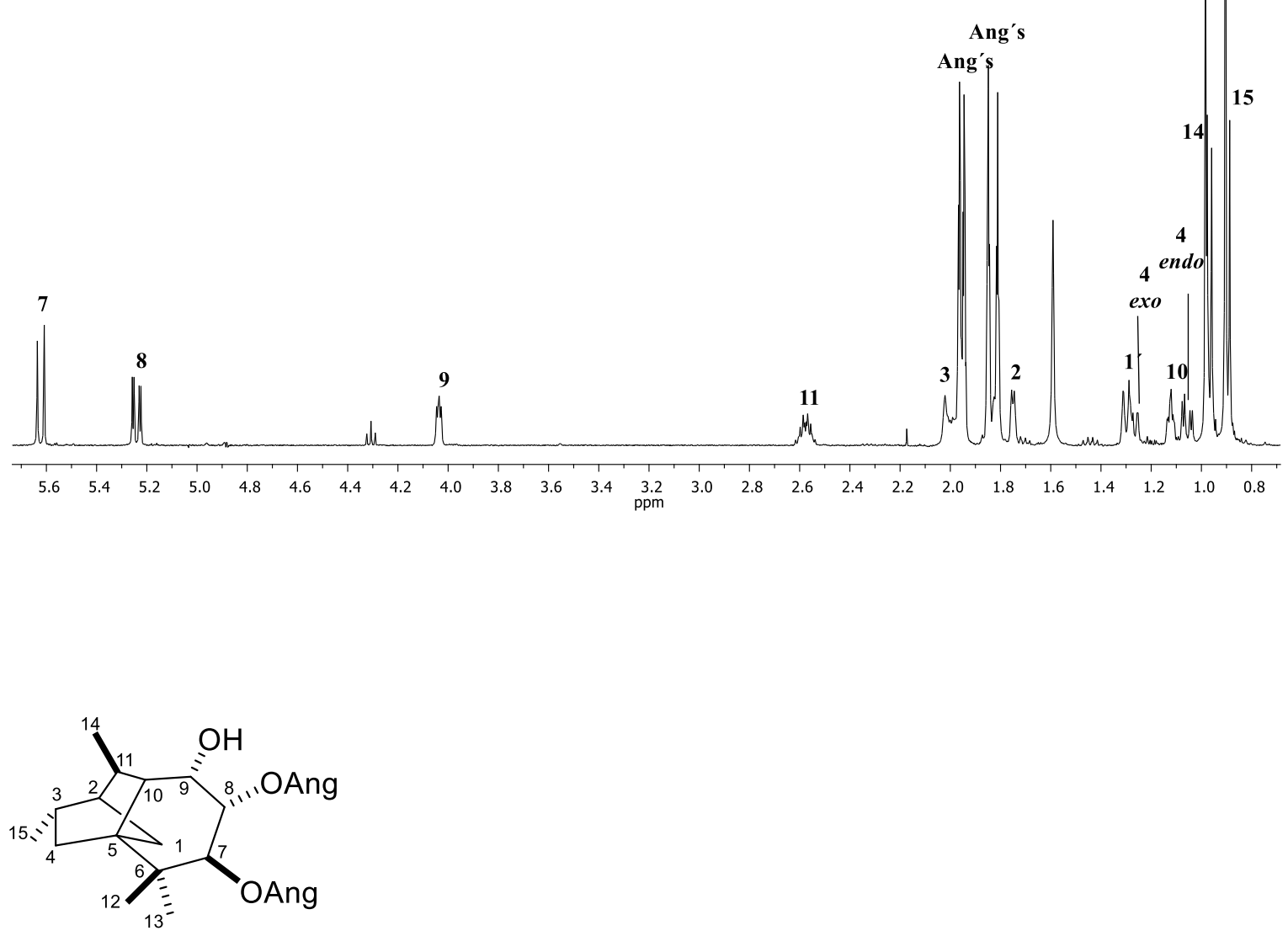

19

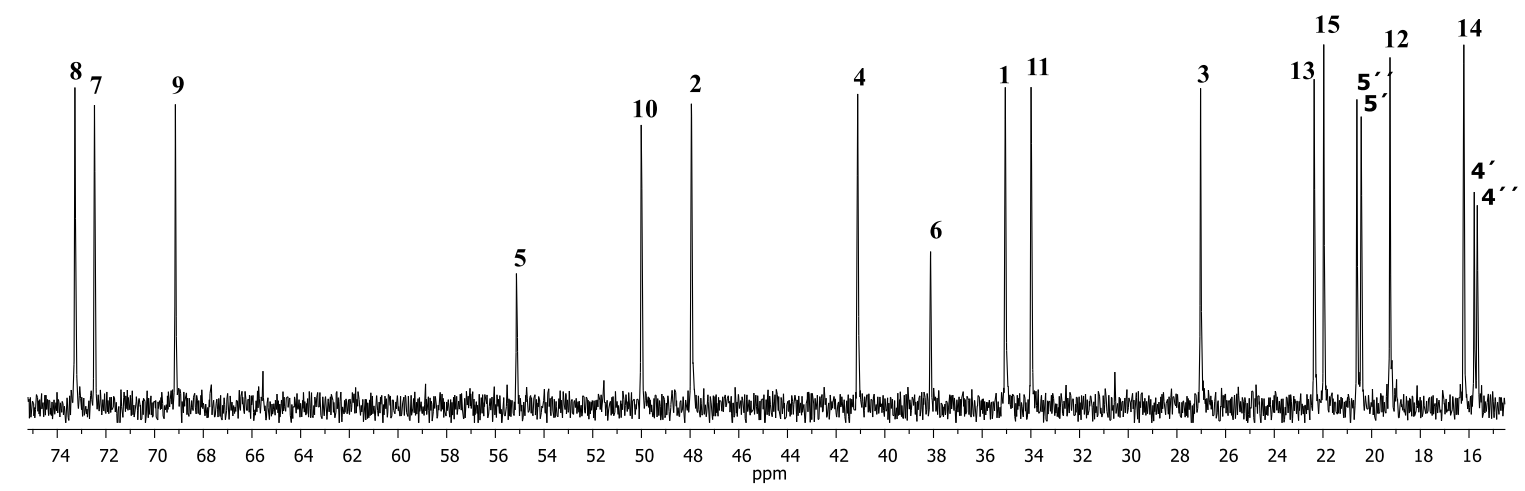

Figure S16. ${ }^{1} \mathrm{H}(400 \mathrm{MHz})$ and ${ }^{13} \mathrm{C}(100 \mathrm{MHz}) \mathrm{NMR}$ spectra of 19 in $\mathrm{CDCl}_{3}$. 


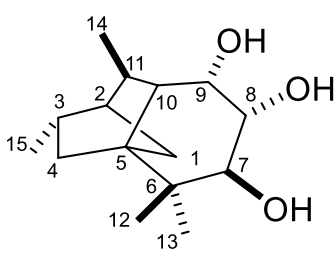

20

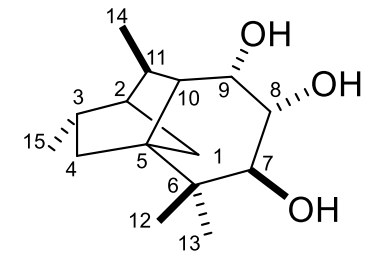

20

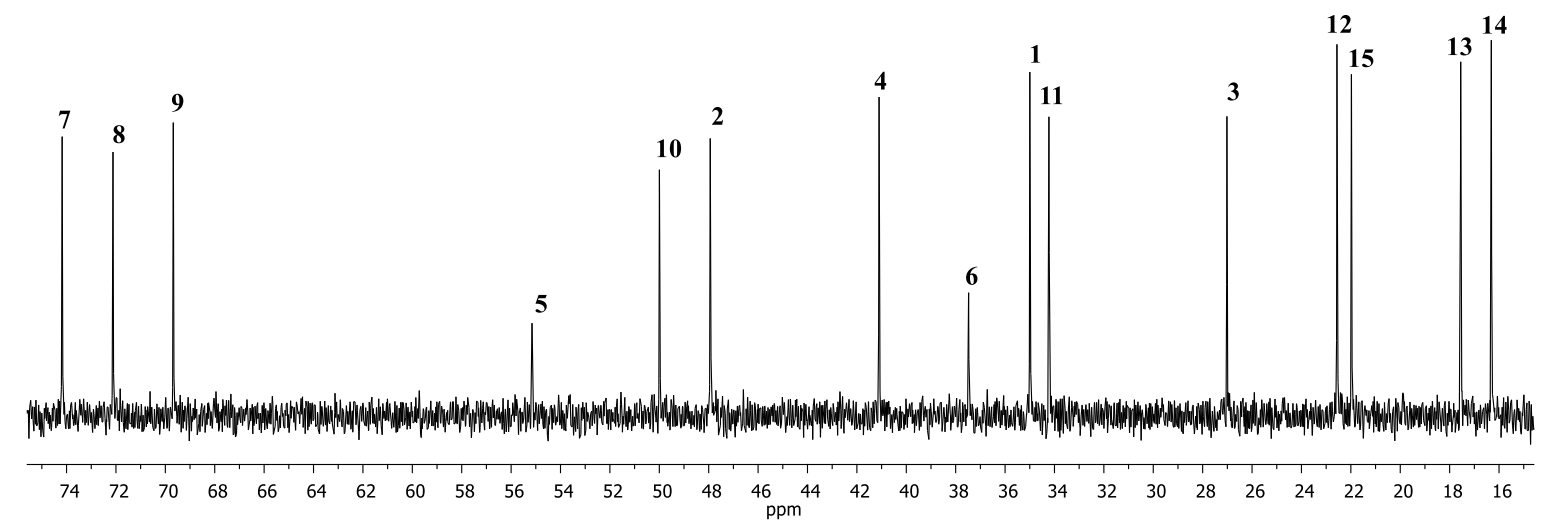

Figure S17. ${ }^{1} \mathrm{H}(400 \mathrm{MHz})$ and ${ }^{13} \mathrm{C}(100 \mathrm{MHz}) \mathrm{NMR}$ spectra of 20 in $\mathrm{CDCl}_{3}$. 


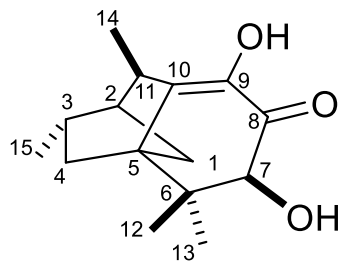

21
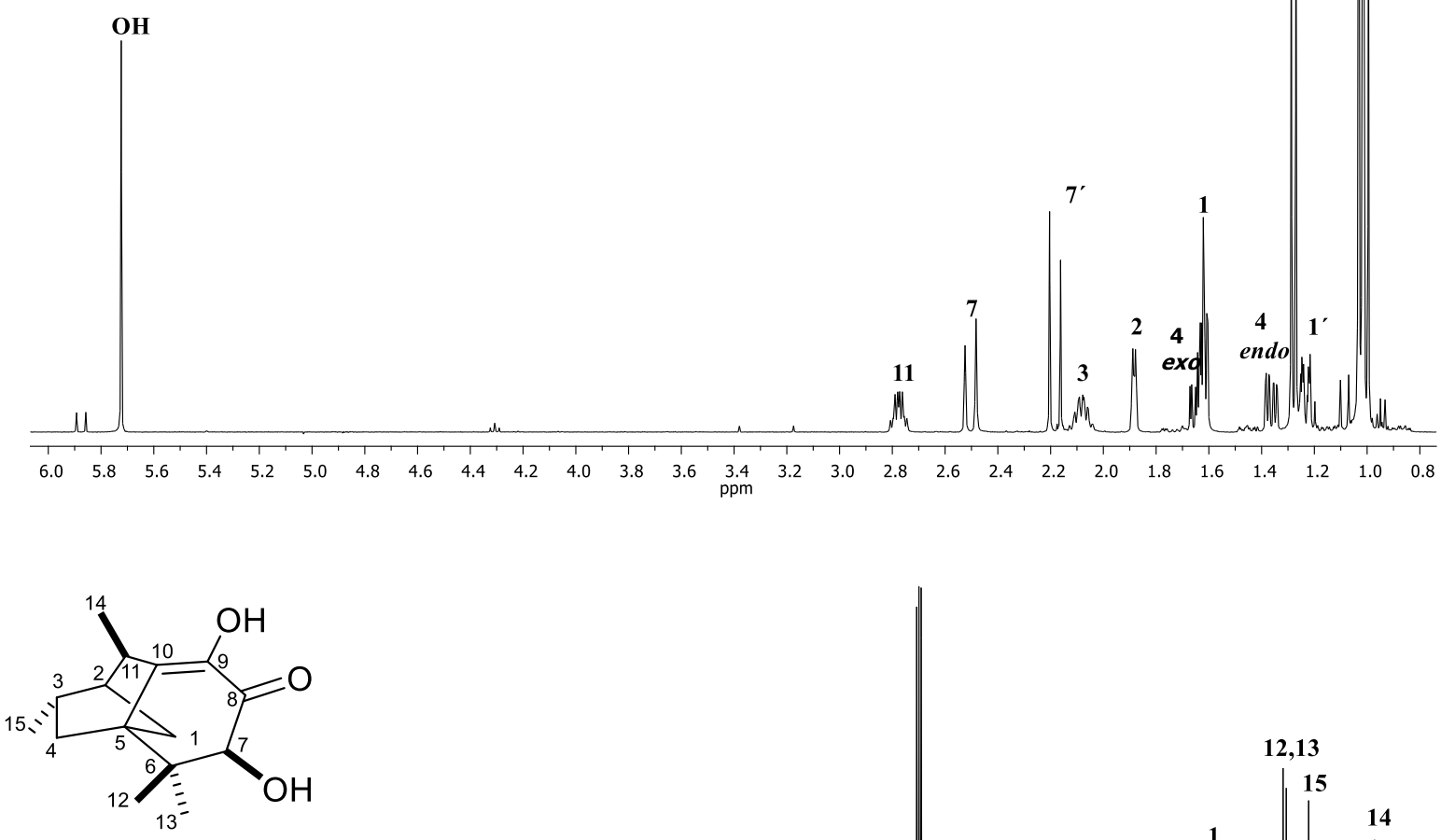

21

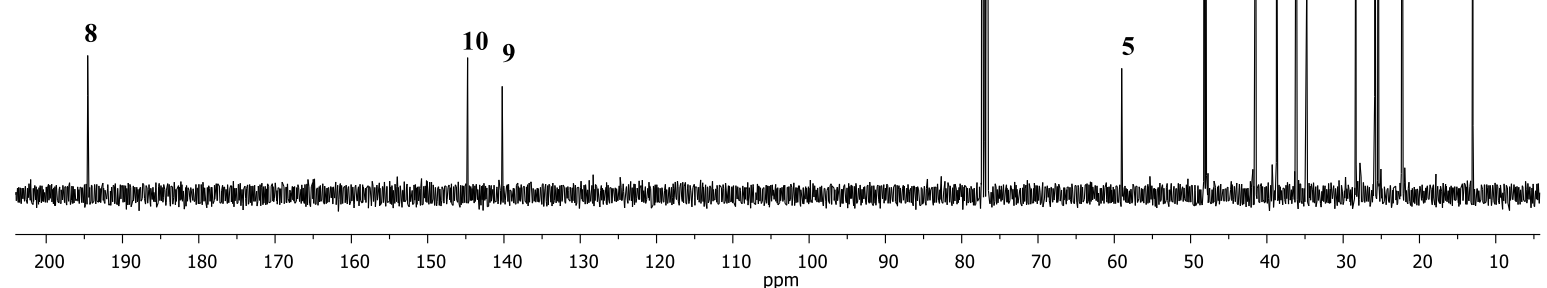

Figure S18. ${ }^{1} \mathrm{H}(400 \mathrm{MHz})$ and ${ }^{13} \mathrm{C}(100 \mathrm{MHz}) \mathrm{NMR}$ spectra of 21 in $\mathrm{CDCl}_{3}$. 


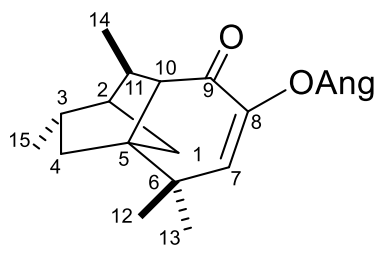

22
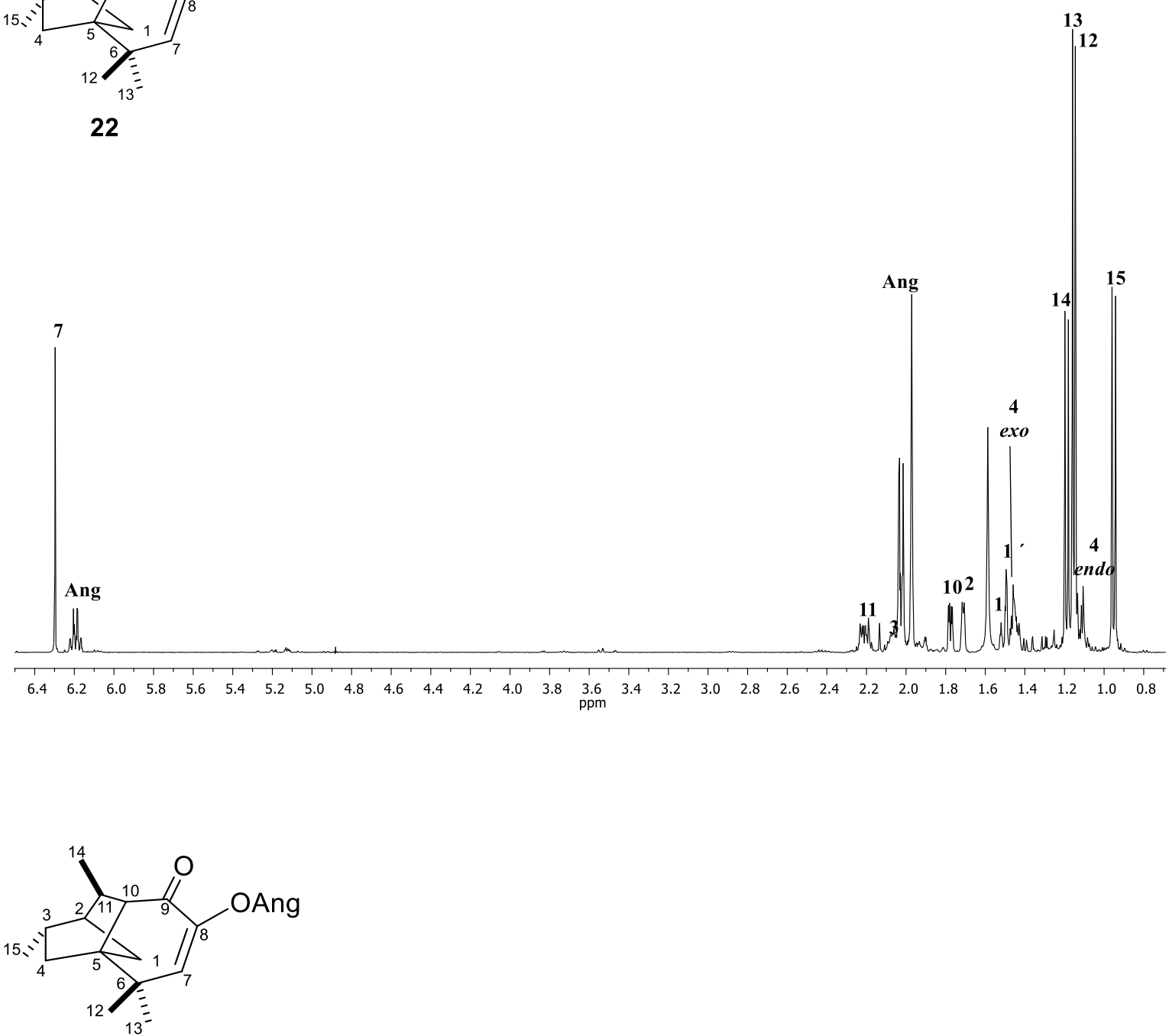

22

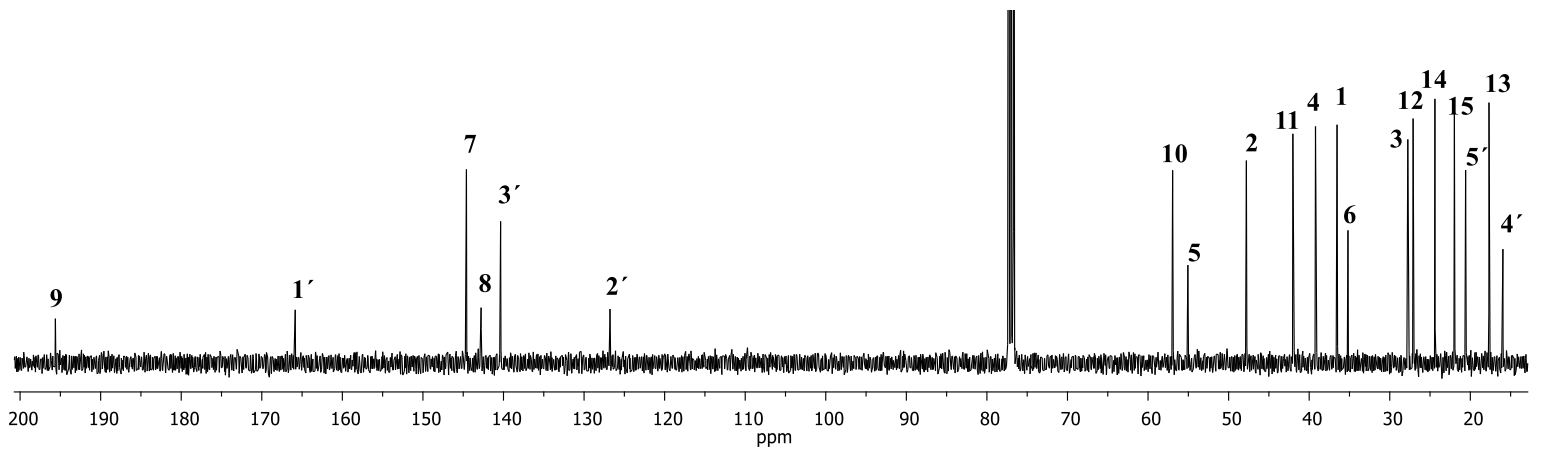

Figure S19. ${ }^{1} \mathrm{H}(400 \mathrm{MHz})$ and ${ }^{13} \mathrm{C}(100 \mathrm{MHz}) \mathrm{NMR}$ spectra of 22 in $\mathrm{CDCl}_{3}$. 
Table S1. Single-Crystal X-ray Data for Compounds 10, 15, 20, and 21

\begin{tabular}{|c|c|c|c|c|}
\hline & 10 & 15 & 20 & 21 \\
\hline Empirical formula & $\mathrm{C}_{22} \mathrm{H}_{30} \mathrm{O}_{5} \mathrm{~S}$ & $\mathrm{C}_{15} \mathrm{H}_{24} \mathrm{O}_{3}$ & $\mathrm{C}_{15} \mathrm{H}_{26} \mathrm{O}_{3}$ & $\mathrm{C}_{15} \mathrm{H}_{22} \mathrm{O}_{2}$ \\
\hline Formula weight & 406.52 & 252.34 & 254.36 & 234.32 \\
\hline Crystal size (mm) & $0.70 \times 0.05 \times 0.04$ & $0.50 \times 0.10 \times 0.10$ & $0.08 \times 0.04 \times 0.04$ & $0.62 \times 0.25 \times 0.25$ \\
\hline Crystal system & monoclinic & orthorhombic & monoclinic & orthorhombic \\
\hline space group & $P 2_{1}$ & $P 2_{1} 2_{1} 2_{1}$ & $P 2_{1}$ & $P 2_{1} 2_{1} 2_{1}$ \\
\hline Cell $a(\breve{A})$ & $9.8062(5)$ & $6.5313(4)$ & $13.668(2)$ & $6.888(1)$ \\
\hline$b(\check{\mathrm{A}})$ & $8.6257(5)$ & $13.2992(5)$ & $7.918(1)$ & $11.405(2)$ \\
\hline$c(\breve{\mathrm{A}})$ & $13.2438(9)$ & $15.6196(7)$ & $15.334(2)$ & $17.139(3)$ \\
\hline$\beta(\mathrm{deg})$ & $108.563(6)$ & 90 & $107.502(8)$ & 90 \\
\hline Volume $\left(\breve{A}^{3}\right)$ & $1061.9(1)$ & $1356.7(1)$ & $1582.7(4)$ & $1346.4(5)$ \\
\hline$Z, \rho_{\text {calc }}\left(\mathrm{mg} / \mathrm{mm}^{3}\right)$ & $2,1.271$ & $4,1.235$ & $4,1.067$ & $4,1.156$ \\
\hline$\mu\left(\mathrm{mm}^{-1}\right)$ & 1.60 & 0.67 & 0.58 & 0.59 \\
\hline $\mathrm{F}(000)$ & 436 & 552 & 560 & 512 \\
\hline Theta range (deg) & 3.5 to 77.6 & 4.4 to 77.87 & 6.5 to 40.09 & 8.2 to 74.5 \\
\hline Reflections collected & 14386 & 19814 & 22250 & 44853 \\
\hline Reflections unique & 4004 & 2865 & 1919 & 2673 \\
\hline Data / parameters & $3224 / 294$ & $2437 / 200$ & $1879 / 353$ & $2595 / 174$ \\
\hline Goodness-of-fit & 1.04 & 1.08 & 1.05 & 1.05 \\
\hline Final R1 (\%) & 3.9 & 4.1 & 3.3 & 3.8 \\
\hline wR2 (\%) & 8.5 & 10.1 & 8.7 & 10.0 \\
\hline Residual $\mathrm{e}^{-}\left(\mathrm{e} . \check{A}^{3}\right)$ & 0.15 and -0.16 & 0.15 and -0.12 & 0.10 and -0.14 & 0.10 and -0.09 \\
\hline $\begin{array}{l}\text { Flack and Hooft } \\
\text { parameters }\end{array}$ & $\begin{array}{l}-0.02(2) \text { and } \\
-0.015(17)\end{array}$ & $\begin{array}{l}0.0(3) \text { and } \\
-0.06(14)\end{array}$ & $\begin{array}{l}-0.16(12) \\
-0.10(12)\end{array}$ & $\begin{array}{l}-0.0(3) \text { and } \\
-0.03(5)\end{array}$ \\
\hline $\begin{array}{l}\text { Inverted Flack and } \\
\text { Hooft parameters }\end{array}$ & $\begin{array}{l}0.91(3) \text { and } \\
1.015(17)\end{array}$ & $\begin{array}{l}1.0(3) \text { and } \\
1.06(14)\end{array}$ & $\begin{array}{l}1.17(12) \\
1.11(11)\end{array}$ & $\begin{array}{l}1.0(3) \text { and } \\
1.03(5)\end{array}$ \\
\hline CCDC deposit No. & 1959728 & 1959729 & 1959730 & 1959731 \\
\hline
\end{tabular}

